\title{
ROBUST ERROR ESTIMATES FOR STABILIZED FINITE ELEMENT APPROXIMATIONS OF THE TWO DIMENSIONAL NAVIER-STOKES' EQUATIONS AT HIGH REYNOLDS NUMBER
}

\author{
ERIK BURMAN*
}

\begin{abstract}
We consider error estimates for stabilized finite element approximations of the twodimensional Navier-Stokes' equations on the unit square with periodic boundary conditions. The estimates for the vorticity are obtained in a weak norm that can be related to the norms of filtered quantities. $L^{2}$-norm estimates are obtained for the velocities. Under the assumption of the existence of a certain decomposition of the solution, into large eddies and small fine scale fluctuations, the constants of the estimates are proven to be independent of the Reynolds number. Instead they depend on the $L^{\infty}$-norm of the initial vorticity and an exponential with factor proportional to the $L^{\infty}$-norm of the gradient of the large eddies. The main error estimates are on a posteriori form, but for certain stabilized methods the residuals may be upper bounded uniformly, leading to robust a priori error estimates.
\end{abstract}

Key words. Navier-Stokes' equations, stability, error estimates, high Reynolds number, finite element methods, stabilization, large eddies

AMS subject classifications. 65M12, 65M60, 76F65

1. Introduction. It is well known that provided the exact solution is sufficiently smooth the approximate solution $u_{h}$ of the Navier-Stokes' equations on velocitypressure form can be proved to satisfy estimates of the type

$$
\left\|\left(u-u_{h}\right)(\cdot, T)\right\|_{L^{2}(\Omega)} \lesssim e^{\|\nabla u\|_{L^{\infty}(Q)} h^{\frac{3}{2}}}|u|_{L^{2}\left(0, T ; H^{2}(\Omega)\right)},
$$

if a consistent stabilized finite element method with piecewise affine approximation is used. Here $u$ denotes the exact solution, $h$ a meshsize, $Q:=\Omega \times I$ a space time domain with $\Omega$ the space domain and $I:=(0, T)$ a time interval with the final time $T$. We use the notation $a \lesssim b$ for $a \leq C b$ with $C$ a constant independent of the physcial parameters of the problem and the mesh size. We will also use $a \sim b$ for $a \lesssim b$ and $b \lesssim a$. We refer to $[12,6]$ for examples of analyses of Navier-Stokes' equations on velocity-pressure form and to $[17,18]$ for analyses on vorticity-streamfunction form. We also give a proof of (1.1) for one of the methods proposed herein in appendix.

Note that there is no explicit dependence on the viscosity in the estimate (1.1). However, for this estimate to be useful the included Sobolev norms must be small, which rarely is the case in the high Reynolds number regime and hence the dependence of the viscosity enters in an implicit manner. If we relax the constraint that the estimate may have no explicit dependence on the viscosity the exponential can be replaced by an exponential of the type $e^{\sqrt{R e} T}$, which blows up for vanishing viscosity. The purpose of the present paper is to prove robust error estimates for a subclass of solutions that satisfy a special scale separation property. This is motivated by the results of [11], where it is shown theoretically that the amplitude of the small structures of the flow are exponentially damped for two-dimensional flows. It would therefore seem reasonable that the constant in the estimate (1.1) could be made to depend only on the gradient of the large scales of the flow, since the fine scales are controlled by viscous dissipation. There is still a dependence on the viscosity entering through the scale separation, since as the viscosity goes to zero, so does the energy

${ }^{*}$ Department of Mathematics, University College London, Gower Street, London, UK-WC1E 6BT, United Kingdom; (e.burman@ucl.ac.uk) 
density of the fine scale component. It appears to be impossible to eliminate this dependence completely, at least if the error is measured in a Sobolev norm, due to the lack of uniform continuous dependence on initial data for the limit case of the incompressible Euler equations (see [13]). In the case of the incompressible Euler equations we get error estimates under the sole assumption that the velocity gradient is pointwise bounded.

Our estimates are based on the following three points:

1. the use of a weak norm for the vorticity estimate;

2. stability estimates for an associated dual problem;

3. the use of a stabilized finite element method, giving enhanced a priori control of residual quantities in the high Reynolds number regime.

The idea of measuring error in filtered quantities was considered in $[9,10]$, but the estimates were not robust in the Reynolds number and the constant included high order Sobolev norms of the exact solution.

In [3] parametrised weak norm estimates were used in order to derive robust error estimates for the transient advection-diffusion equation under the assumption that the transport velocity satisfies a certain scale separation hypothesis that we will discuss in detail below.

To define the subclass of solutions for which our estimates are robust we assume that there are relatively smooth eddies, with large associated Reynolds number, containing the bulk of the energy and small scale fluctuations that may vary rapidly in space, but carry a negligible part of the energy. In other words we assume that all the scales of the flow are subject to a global time scale $\tau_{F}$, that is set by the large scales and therefore moderately small, and that the fine scale Reynolds number is $O(1)$.

To make this precise, we consider the following decomposition of the exact solution in the large scales $\bar{u}$ and a rough fine scale $u^{\prime}$, with small energy. Indeed for any $t \in I$ and for $\epsilon \sim \nu^{\frac{1}{2}}$ we write

$$
u=\bar{u}+u^{\prime}, \quad \bar{u} \in W^{1, \infty}(\Omega), u^{\prime} \in W^{1, p}(\Omega) \cap L^{\infty}(\Omega), p>2 \text { and }\left\|u^{\prime}\right\|_{L^{\infty}(\Omega)}<\epsilon
$$

where $\bar{u}$ is periodic on $\Omega$. Robustness of the estimates are obtained if we assume that a decomposition on the form (1.2) exists with $\|\nabla \bar{u}\|_{L^{\infty}(Q)} \sim 1$. To relate the residual energy density $\epsilon^{2}$ to the viscous dissipation we define the following global time scale for the flow in the space-time domain

$$
\tau_{F}:=\|\nabla \bar{u}\|_{L^{\infty}(Q)}^{-1} .
$$

It follows that for smooth large scales $\tau_{F} \sim 1$. Then we assume that the fine scale Reynolds number is one.

$$
R e^{\prime}=\frac{\left\|u^{\prime}\right\|_{L^{\infty}(Q)} l^{\prime}}{\nu}=1
$$

Under the assumption of a global time scale, the length scale $l^{\prime}$ based on $\left\|u^{\prime}\right\|_{L^{\infty}(Q)}$ and $\tau_{F}$ writes

$$
l^{\prime}:=\left\|u^{\prime}\right\|_{L^{\infty}(Q)} \tau_{F}
$$

and we obtain the following relation between the fine scale energy density and the kinematic viscosity,

$$
1=\frac{\left\|u^{\prime}\right\|_{L^{\infty}(Q)} l^{\prime}}{\nu}=\frac{\left\|u^{\prime}\right\|_{L^{\infty}(Q)}^{2} \tau_{F}}{\nu} \rightarrow\left\|u^{\prime}\right\|_{L^{\infty}(Q)}^{2} \leq \nu .
$$


We will prove that independent of $\nu$, the abstract decomposition (1.2) always exists for solutions to the Navier-Stokes' equations in two dimensions and that it allows us to replace $\|\nabla u\|_{L^{\infty}(Q)}$ in the exponential by $\|\nabla \bar{u}\|_{L^{\infty}(Q)}$. Of course we do not claim that all flows allow for a decomposition with $\|\nabla \bar{u}\|_{L^{\infty}(Q)}$ moderate. Counter examples, that are sensitive to perturbations in initial data, are easily found, such as Kelvin-Helmholtz shear flows and we will show some numerical experiments indicating that the exponential with gradient dependent factor is indeed relevant. How much the exponential factor can be reduced using scale separation depends on the particular flow. It is also worth noting that the below analysis uses the two dimensional setting in several ways. In three space dimensions the appearance of the vortex stretching term makes it impossible to absorb the fine scale velocities using the viscous dissipation and the regularity of the exact solution is insufficient for the scale separation construction. There is no longer a maximum principle for the vorticity and the Sobolev injection used in the finite element analysis, that is dimension dependent, no longer leads to a converging method.

We prove error estimates on the vorticity in a weak norm, that will be specified below, these estimates may then be used to obtain the following bound for the flow velocities,

$$
\sup _{t \in(0, T)}\left\|u-u_{h}\right\|_{L^{2}(\Omega)} \leq C_{0}\left(\omega_{0}, T\right) e^{\frac{T}{\tau_{F}}} h^{\frac{1}{2}}
$$

The constant $C_{0}$ depends only on the initial vorticity, time and the mesh geometry, with at most linear growth in time. For solutions that have the scale separation property discussed above $\tau_{F} \sim 1$ and the computation is robust even in the presence of rough velocity fluctuations with small amplitude.

Herein our main concern will be the high mesh Reynolds number case

$$
R e_{h}:=\frac{U_{0} h}{\nu}>1
$$

where $U_{0}:=\|u(\cdot, 0)\|_{L^{\infty}(\Omega)} \sim 1$ denotes the characteristic velocity of the flow, but many results are independent of the mesh Reynolds number. It will always be explicitly stated when a result only holds in the high Reynolds number regime. If the local Reynolds number is low, other approaches than those presented herein might be more appropriate.

For the discretization we will use a finite element method with stabilization to handle the high Reynolds number regime. We will consider both linear methods of artificial viscosity type restricted to first order accuracy and weakly consistent stabilization operators allowing for high order accuracy. We will also consider nonlinear stabilization methods of shock capturing type, providing new error estimates for such methods.

2. The Navier-Stokes' equations in two space dimensions. Let $\Omega$ be the unit square and define $H_{\text {per }}^{1}(\Omega)$ to be the space of functions in $H^{1}(\Omega)$ that are periodic in both cartesian directions. The $L^{2}$-scalar product over some space or space-time domain $X$ will be denoted $(\cdot, \cdot)_{X}$ with associated norm $\|\cdot\|_{X}$ where the subscript may be dropped for $X=\Omega$. Denoting by $\omega$ the vorticity of the flow and by $\Psi$ the 
streamfunction, the equations may be written

$$
\begin{aligned}
\partial_{t} \omega+u \cdot \nabla \omega-\nu \Delta \omega & =0, \text { in } Q, \\
-\Delta \Psi & =\omega \text { in } Q, \\
u & =\operatorname{rot} \Psi \text { in } Q, \\
\omega(x, 0) & =\omega_{0},
\end{aligned}
$$

with $\omega_{0} \in L^{\infty}(\Omega)$. Let $L_{*}:=\left\{q \in L^{2}(\Omega) ; \int_{\Omega} q=0\right\}$. Continuity of normal fluxes of $\omega$ and $\Psi$ over the periodic boundaries are also imposed. The associated weak formulation takes the form, for $t>0$, find $(\omega, \Psi) \in H_{\text {per }}^{1}(\Omega) \times H_{p e r}^{1}(\Omega) \cap L_{*}(\Omega)$ such that

$$
\begin{aligned}
\left(\partial_{t} \omega, v\right)_{\Omega}+a(u ; \omega, v) & =0, \\
(\nabla \Psi, \nabla \Phi)_{\Omega} & =(\omega, \Phi)_{\Omega}, \\
u & =\operatorname{rot} \Psi,
\end{aligned}
$$

for all $(v, \Phi) \in H_{p e r}^{1}(\Omega) \times H_{p e r}^{1}(\Omega) \cap L_{*}(\Omega)$, where the semi-linear form $a(\cdot ; \cdot, \cdot)$ is defined by

$$
a(u ; \omega, v):=(u \cdot \nabla \omega, v)_{\Omega}+(\nu \nabla \omega, \nabla v)_{\Omega} .
$$

This problem is known to be well-posed, but a priori error estimates on the solution are in general strongly dependent on the viscosity coefficient reflecting the poor stability of the equations in the high Reynolds number regime. Note however that the solution of (2.2)-(2.3), will have a decomposition of the form (1.2), although no quantitative estimate seems to be obtainable for $\|\nabla \bar{u}\|_{L^{\infty}(\Omega)}$ in terms of data.

LEMMA 2.1. Let $u$ be the solution of (2.1). Then, for all $\epsilon>0$, the decomposition defined by (1.2) exists independently of $\nu$.

Proof. Since $u=\operatorname{rot} \Psi$, where $\Psi$ solves $-\Delta \Psi=\omega$, by elliptic regularity and the maximum principle there holds, for all $1<p<\infty,\|u\|_{W^{1, p}(\Omega)} \lesssim\|\|_{L^{p}(\Omega)} \lesssim$ $\left\|\omega_{0}\right\|_{L^{\infty}(\Omega)}$. By Sobolev injection for $p>2$ we have $u \in C^{0}(\bar{\Omega})$. We may then use a standard mollifier $\phi_{\epsilon}$ to define $\bar{u}:=\phi_{\epsilon} * u \in C^{\infty}(\bar{\Omega})$ so that $\left\|u^{\prime}\right\|_{L^{\infty}(\Omega)}=$ $\left\|u-\phi_{\epsilon} * u\right\|_{L^{\infty}(\Omega)}<\epsilon$.

3. Finite element discretization. Let $\left\{\mathcal{T}_{h}\right\}_{h>0}$ be a family of affine, shape regular (in the sense of [1]) meshes on $\Omega$. We assume that the meshes are kept fixed in time. The index $h$ is defined as the maximum diameter $h_{K}$ of any triangle $K$ in $\mathcal{T}_{h}, h=\max _{K \in \mathcal{T}_{h}} h_{K}$. Mesh faces are collected in the set $\mathcal{F}$ and we denote the length of a face by $h_{F}$. For a smooth enough function $v$ that is possibly double-valued at $F \in \mathcal{F}$ with $F=\partial K^{-} \cap \partial K^{+}$, we define its jump at $F$ as $\llbracket v \rrbracket:=\left.v\right|_{K^{-}}-\left.v\right|_{K^{+}}$, and we fix the unit normal vector to $F$, denoted by $n_{F}$, as pointing from $K^{-}$to $K^{+}$. The arbitrariness in the sign of $\llbracket v \rrbracket$ is irrelevant in what follows. Let $P_{k}(K)$ denote the set of polynomials of degree less than or equal to $k$ on the simplex $K$. Define $V_{h}^{k}$ to be the standard space of piecewise polynomial, continuous periodic functions,

$$
V_{h}^{k}:=\left\{v_{h} \in C_{p e r}^{0}(\bar{\Omega}):\left.v_{h}\right|_{K} \in P_{k}(K) ; \forall K \in \mathcal{T}_{h}\right\} .
$$

We let $\pi_{L}$ denote the $L^{2}$-projection on $V_{h}^{k}$ and $\pi_{V}$ the $H^{1}$-projection on $V_{h}^{k}$

$$
\left(\nabla \pi_{V} u, \nabla v_{h}\right)_{\Omega}=\left(\nabla u, \nabla v_{h}\right)_{\Omega} \quad \forall v_{h} \in V_{h} \text { and } \int_{\Omega}\left(\pi_{V} u-u\right) \mathrm{d} x=0
$$


We recall that if $u \in H^{r}(\Omega), r \geq 1$, the following approximation estimates hold for $\pi_{L}$ and $\pi_{V}$

$$
\left\|h^{-1}\left(\pi_{L} u-u_{h}\right)\right\|+\left\|\nabla\left(\pi_{L} u-u\right)\right\| \leq c_{0} h^{s-1}|u|_{H^{s}(\Omega)}, \text { with } s=\min (k+1, r)
$$

and

$$
\left\|h^{-1}\left(\pi_{V} u-u_{h}\right)\right\|+\left\|\nabla\left(\pi_{V} u-u\right)\right\| \leq c_{1} h^{s-1}|u|_{H^{s}(\Omega)}, \text { with } s=\min (k+1, r) .
$$

Observe that we here used the $H^{1}$-stability of the $L^{2}$-projection valid on shape regular meshes (see [1]). We consider continuous finite elements with $k=1$ to discretize the vorticity $\omega$ in space and $k=1,2$ for the stream function $\Psi$. The discrete velocity is given element wise by $\left.u_{h}\right|_{K}:=\operatorname{rot} \Psi_{h}:=\left(\partial_{y} \Psi_{h},-\partial_{x} \Psi_{h}\right)$. Note that using this definition $\nabla \cdot u_{h}=0$ in $\Omega$, i.e. the discrete velocity is globally divergence free. We discretise in space using a stabilized finite element method. For $t>0$ find $\left(\omega_{h}, \Psi_{h}\right) \in$ $V_{h}^{1} \times V_{*}^{l}$, with $l=1,2$, such that

$$
\begin{aligned}
m\left(\partial_{t} \omega_{h}, v_{h}\right)+a\left(u_{h} ; \omega_{h}, v_{h}\right)+s\left(u_{h} ; \omega_{h}, v_{h}\right) & =0, \\
\left(\nabla \Psi_{h}, \nabla \Phi_{h}\right)_{\Omega}-\left(\omega_{h}, \Phi_{h}\right)_{\Omega} & =0, \\
u_{h}-\operatorname{rot} \Psi_{h} & =0,
\end{aligned}
$$

for all $\left(v_{h}, \Phi_{h}\right) \in V_{h}^{1} \times V_{*}^{l}$ and with initial data $w_{0}:=\pi_{L} \omega(\cdot, 0)$. Here $s(\cdot ; \cdot, \cdot)$ denotes a stabilization operator that is linear in its last argument and $m(\cdot, \cdot)$ denotes the bilinear form defining the mass matrix, this operator either coincides with $(\cdot, \cdot)_{\Omega}$ or is evaluated using nodal quadrature, i.e. so called mass lumping. In the latter case we have

$$
m\left(u_{h}, v_{h}\right):=\int_{\Omega} i_{h}\left(u_{h} v_{h}\right) \mathrm{d} x,
$$

where $i_{h}$ denotes the nodal interpolant on $V_{h}^{1}$. We will assume that the stabilization term satisfies

$U_{0}^{-\frac{1}{2}} \inf _{v_{h} \in V_{h}^{1}}\left\|h^{\frac{1}{2}}\left(u_{h} \cdot \nabla \omega_{h}-v_{h}\right)\right\| \lesssim s\left(u_{h} ; \omega_{h}, \omega_{h}\right)^{\frac{1}{2}} \lesssim h^{\frac{1}{2}}\left(U_{0}^{\frac{1}{2}}+U_{0}^{-\frac{1}{2}}\left\|u_{h}\right\|_{L^{\infty}(\Omega)}\right)\left\|\nabla \omega_{h}\right\|$

and

$$
s\left(u_{h} ; \omega_{h}, v_{h}\right) \lesssim h^{\frac{1}{2}}\left(U_{0}^{\frac{1}{2}}+U_{0}^{-\frac{1}{2}}\left\|u_{h}\right\|_{L^{\infty}(\Omega)}\right) s\left(u_{h} ; \omega_{h}, \omega_{h}\right)^{\frac{1}{2}}\left\|\nabla v_{h}\right\| .
$$

LEMmA 3.1. The following estimates hold for the solution of (3.3)-(3.4).

$$
\begin{gathered}
\sup _{t \in I}\left\|\omega_{h}(\cdot, t)\right\|^{2}+2\left\|\nu^{\frac{1}{2}} \nabla \omega_{h}\right\|_{Q}^{2}+2 \int_{I} s\left(u_{h} ; \omega_{h}, \omega_{h}\right) d t \lesssim\|\omega(\cdot, 0)\|^{2}, \\
\left\|u_{h}(\cdot, t)\right\|_{L^{\infty}(\Omega)} \leq c_{q}\left\|\omega_{h}(\cdot, t)\right\|_{L^{q}(\Omega)}, \quad q>2 .
\end{gathered}
$$

If $l=1$ and the stabilization operator $s\left(u_{h} ; \omega_{h}, v_{h}\right)$ satisfies (3.5) and (3.6) then,

$$
\int_{I}\left\|\nabla \partial_{t} \omega_{h}\right\| d t \lesssim \int_{I}\left(h^{-\frac{3}{2}}\left(U_{0}^{\frac{1}{2}}+U_{0}^{\frac{1}{2}}\left\|u_{h}(\cdot, t)\right\|_{L^{\infty}(\Omega)}\right) s\left(u_{h} ; \omega_{h}, \omega_{h}\right)^{\frac{1}{2}}+\nu h^{-2}\left\|\nabla \omega_{h}\right\|\right) d t .
$$


Proof. Observe that by norm equivalence on discrete spaces the $L^{2}$-norm defined using nodal quadrature is equivalent to the standard $L^{2}$-norm. Then the inequality (3.7) is immediate by taking $v_{h}=\omega_{h}$ in (3.3), integrating and using the stability of the $L^{2}$-projection. For the inequality $(3.8)$, consider the auxiliary problem, $-\Delta \tilde{\Psi}=\omega_{h}$ in $\Omega$ and note that by [20] (and [8] for the case $l=2$ ), there holds

$$
\left\|u_{h}(\cdot, t)\right\|_{L^{\infty}(\Omega)} \lesssim\|\tilde{\Psi}(\cdot, t)\|_{W^{1, \infty}(\Omega)}
$$

and adapting the analysis of [19] we have for the (simpler) case of periodic boundary conditions,

$$
\|\tilde{\Psi}(\cdot, t)\|_{W^{1, \infty}(\Omega)} \leq c_{q}\left\|\omega_{h}(\cdot, t)\right\|_{L^{q}(\Omega)}, q>2 .
$$

To prove (3.9) finally we introduce a function $\xi_{h} \in V_{h}^{1}$ such that

$$
m\left(\xi_{h}, v_{h}\right)=\left(\nabla \partial_{t} \omega_{h}, \nabla v_{h}\right)_{\Omega}, \quad \forall v_{h} \in V_{h}^{1} .
$$

It follows by taking $v_{h}=\xi_{h}$ and using the Cauchy-Schwarz inequality followed by an inverse inequality that

$$
m\left(\xi_{h}, \xi_{h}\right)^{\frac{1}{2}} \sim\left\|\xi_{h}\right\| \lesssim h^{-1}\left\|\partial_{t} \nabla \omega_{h}\right\|
$$

The consistency error of the mass-lumping may be estimated as follows

$$
\begin{aligned}
\left|m\left(v_{h}, \xi_{h}\right)-\left(v_{h}, \xi_{h}\right)_{\Omega}\right|= & \left|\int_{\Omega}\left(i_{h}\left(v_{h} \xi_{h}\right)-v_{h} \xi_{h}\right) \mathrm{d} x\right| \\
& \lesssim \sum_{K \in \mathcal{T}_{h}} \int_{K} h^{2}\left|D^{2}\left(v_{h} \xi_{h}\right)\right| \mathrm{d} x \lesssim\left(h^{2}\left|\nabla v_{h}\right|,\left|\nabla \xi_{h}\right|\right)_{\Omega} .
\end{aligned}
$$

Taking $v_{h}=\xi_{h}$ in $(3.3)$ yields

$$
\left\|\partial_{t} \nabla \omega_{h}\right\|^{2}=-\left(u_{h} \cdot \nabla \omega_{h}, \xi_{h}\right)_{\Omega}-\left(\nu \nabla \omega_{h}, \nabla \xi_{h}\right)_{\Omega}-s\left(u_{h} ; \omega_{h}, \xi_{h}\right) .
$$

We may then apply the Cauchy-Schwarz inequality in the second term of the right hand side of (3.12) and (3.6) in the last term, followed by inverse inequalities on $\left\|\nabla \xi_{h}\right\|$ and the estimate (3.10). For the first term in the right hand side of (3.12) we obtain, by adding and subtracting $m\left(v_{h}, \xi_{h}\right)-\left(v_{h}, \xi_{h}\right)_{\Omega}$, for any $v_{h} \in V_{h}^{1}$, using the properties of $\xi_{h}$ and the bound (3.11)

$$
\left|\left(u_{h} \cdot \nabla \omega_{h}, \xi_{h}\right)_{\Omega}\right| \lesssim\left|\left(u_{h} \cdot \nabla \omega_{h}-v_{h}, \xi_{h}\right)_{\Omega}\right|+\left|\left(\partial_{t} \nabla \omega_{h}, \nabla v_{h}\right)_{\Omega}\right|+\left(h^{2}\left|\nabla v_{h}\right|,\left|\nabla \xi_{h}\right|\right)_{\Omega} .
$$

Since both $u_{h}$ and $\nabla \omega_{h}$ are constant per element $\left.\nabla v_{h}\right|_{K}=\left.\nabla\left(v_{h}-u_{h} \cdot \nabla \omega_{h}\right)\right|_{K}$. Using inverse inequalities and the bound (3.10) on $\xi_{h}$ we have

$$
\begin{aligned}
\left|\left(u_{h} \cdot \nabla \omega_{h}, \xi_{h}\right)_{\Omega}\right| \lesssim\left|\left(u_{h} \cdot \nabla \omega_{h}-v_{h}, \xi_{h}\right)_{\Omega}\right| & +\left|\left(\partial_{t} \nabla \omega_{h}, \nabla v_{h}\right)_{\Omega}\right|+\left(h^{2}\left|\nabla v_{h}\right|,\left|\nabla \xi_{h}\right|\right)_{\Omega} \\
& \lesssim h^{-1}\left\|\partial_{t} \nabla \omega_{h}\right\|\left\|u_{h} \cdot \nabla \omega_{h}-v_{h}\right\| .
\end{aligned}
$$

The bound (3.9) now follows from (3.12) using the bounds (3.13), (3.5), (3.10) and finally integrating in time.

Taking the difference of the formulations $(2.2)-(2.3)$ (with $v=v_{h}$ ) and (3.3) - (3.4) 
and setting $e_{\omega}=\omega-\omega_{h}$ and $e_{\Psi}=\Psi-\Psi_{h}$, the following consistency relation holds for all $t>0$,

$$
\begin{aligned}
\left(\partial_{t} e_{\omega}+u \cdot \nabla e_{\omega}+\operatorname{rot} e_{\Psi} \cdot \nabla \omega_{h}, v_{h}\right)_{\Omega}+\left(\nu \nabla e_{\omega}, \nabla v_{h}\right)_{\Omega}= & m\left(\partial_{t} \omega_{h}, v_{h}\right)-\left(\partial_{t} \omega_{h}, v_{h}\right)_{\Omega} \\
& +s\left(u_{h} ; \omega_{h} ; v_{h}\right) \\
\left(\nabla e_{\Psi}, \nabla \Phi_{h}\right)_{\Omega}-\left(e_{\omega}, \Phi_{h}\right)_{\Omega}= & 0 .
\end{aligned}
$$

As mentioned in the introduction, if the solution $(u, \omega)$ is smooth one may prove an error estimate that is robust with respect to $\nu$ using standard linear theory and perturbation arguments. For the methods we consider herein, this result is an extension of the works in [18] and [6] and we state it here only with the dominant terms present. For the readers convenience we briefly outline the proof using one stabilization operator (defined in equation (6.7)) in Appendix A.

Proposition 3.2. Let $(u, \omega)$ be a smooth solution of $(2.2)-(2.3)$ and $\left(u_{h}, \omega_{h}\right)$ be the solution of (3.3)-(3.4), with $m(\cdot, \cdot)=(\cdot, \cdot)_{\Omega}$ and the stabilization term defined by (6.7). Then for $l=1,2$

$$
\begin{aligned}
\left\|\nabla\left(\pi_{V} \Psi-\Psi_{h}\right)(\cdot, T)\right\|+\left\|\left(\pi_{L} \omega-\omega_{h}\right)(\cdot, T)\right\|+\left(\int_{I} s\left(u_{h} ; \omega_{h}, \omega_{h}\right) d t\right)^{\frac{1}{2}} \\
\\
\quad \lesssim c_{\omega}\left(\left(\nu^{\frac{1}{2}}+h^{\frac{1}{2}}\right) h|\omega|_{L^{2}\left(I ; H^{2}(\Omega)\right)}+h^{l}\|\Psi\|_{L^{2}\left(I ; H^{l+1}(\Omega)\right)}\right)
\end{aligned}
$$

where $c_{\omega}:=e^{\|\nabla \omega\|_{L} \infty(Q)} T^{T}$.

Observe that the exponential factor here depends on $\|\nabla \omega\|_{L^{\infty}(Q)}$, compared to $\|\nabla u\|_{L^{\infty}(Q)}$ in (1.1). This is the prize we pay for estimating the $L^{2}$-error of the vorticity.

4. Dual problem. The consistency relation (3.14) suggests the following (homogeneous) perturbation formulation for the evolution of $\left\{e_{\omega}, e_{\Psi}\right\}$

$$
\begin{aligned}
\left(\partial_{t} e_{\omega}+u \cdot \nabla e_{\omega}+\operatorname{rot} e_{\Psi} \cdot \nabla \omega_{h}, \varphi_{1}\right)_{Q}+\left(\nu \nabla e_{\omega}, \nabla \varphi_{1}\right)_{Q} & =0, \\
\left(\nabla e_{\Psi}, \nabla \varphi_{2}\right)_{Q}-\left(e_{\omega}, \varphi_{2}\right)_{Q} & =0,
\end{aligned}
$$

where $\varphi_{1}, \varphi_{2}$ are the solutions to a dual adjoint perturbation equation related to the continuous equation (2.2)-(2.3) and the discretization (3.3)-(3.4). Since the jump of the tangential derivative of $\omega_{h}$ is zero, we may integrate by parts in (4.1), to arrive at the dual adjoint problem

$$
\begin{aligned}
-\partial_{t} \varphi_{1}-u \cdot \nabla \varphi_{1}-\varphi_{2}-\nu \Delta \varphi_{1} & =0 \text { in } Q, \\
-\Delta \varphi_{2}-\nabla \omega_{h} \cdot \operatorname{rot} \varphi_{1} & =0 \text { in } Q, \\
\varphi_{1}(x, T) & =\xi_{0}(x) \text { in } \Omega,
\end{aligned}
$$

where $\xi_{0}(x)$ is some initial data to be fixed later, the choice of $\xi_{0}$ determines the quantity of interest.

A key result for the present analysis is the stability estimate for the dual adjoint solution. The stability constant of this estimate generally takes the form $\exp \left(T / \tau_{F}\right)$ where $\tau_{F}$ denotes a characteristic time scale of the flow. Typically this timescale is determined by the finest scales of the flow and proportional to the inverse of the maximum velocity gradient $\left(\tau_{F}\right)^{-1} \sim\|\nabla u\|_{L^{\infty}(Q)}$. Here we will show that for positive viscosity the finest scales can be absorbed by viscous dissipation leading to the 
following definition of $\tau_{F}$

$$
\left(\tau_{F}\right)^{-1}:=\inf _{\bar{u} \in L^{\infty}\left(I ; W^{1, \infty}(\Omega)\right)}\left\|\sigma_{p}^{+}\left(\Lambda\left(\bar{u}, u^{\prime}, \nu\right)\right)\right\|_{L^{\infty}(Q)}
$$

where $u^{\prime}:=u-\bar{u}, \sigma_{p}^{+}(A)$ denotes the largest positive eigenvalue of the matrix $A$ and $\Lambda\left(\bar{u}, u^{\prime}, \nu\right)$ is a two by two, symmetric matrix defined by,

$$
\Lambda\left(\bar{u}, u^{\prime}, \nu\right)=-\nabla_{S} \bar{u}+\frac{1}{2} \nabla \cdot \bar{u} \mathcal{I}_{2 \times 2}+\frac{1}{2 \nu} u^{\prime \mathrm{T}} u^{\prime},
$$

where $\mathcal{I}_{2 \times 2}$ denotes the identity matrix. It follows that the timescale $\tau_{F}$ now depends on the maximum gradient of the large scales $\bar{u}$ and the amplitude of the fine scales $u^{\prime}$. If the scale separation assumption defined by (1.3) and (1.4) holds with smooth large eddies $\bar{u}$, then $\tau_{F} \sim 1$.

Proposition 4.1. The following stability estimate holds for the solution $\left(\varphi_{1}, \varphi_{2}\right)$ of $(4.2)-(4.4)$,

$$
\begin{array}{r}
\sup _{t \in I}\left\|\nabla \varphi_{1}(\cdot, t)\right\|+\left\|\nu^{\frac{1}{2}} D^{2} \varphi_{1}\right\|_{Q} \lesssim e^{\frac{T}{\tau_{F}}}\left\|\nabla \xi_{0}\right\| \\
\int_{I}\left\|\nabla \varphi_{2}(\cdot, t)\right\| d t \lesssim e^{\frac{T}{T_{F}}} \int_{I}\left\|\omega_{h}\right\|_{L^{\infty}(\Omega)} d t\left\|\nabla \xi_{0}\right\|
\end{array}
$$

where $\tau_{F}$ is defined by equation (4.5) and (4.6).

Proof. First multiply (4.2) by $-\Delta \varphi_{1}$ and (4.3) by $\varphi_{1}$ and integrate over $Q^{*}:=$ $\Omega \times\left(t^{*}, T\right)$, where $t^{*}$ is a time to be chosen. By summing the two relations we obtain

$$
\underbrace{\left(\partial_{t} \varphi_{1}, \Delta \varphi_{1}\right)_{Q^{*}}}_{I_{1}}+\underbrace{\left(u \cdot \nabla \varphi_{1}, \Delta \varphi_{1}\right)_{Q^{*}}}_{I_{2}}+\underbrace{\left(\nabla \omega_{h} \cdot \operatorname{rot} \varphi_{1}, \varphi_{1}\right)_{Q^{*}}}_{I_{3}}+\left\|\nu^{\frac{1}{2}} \Delta \varphi_{1}\right\|_{Q^{*}}^{2}=0 .
$$

We will now treat the terms $I_{1}-I_{3}$ term by term. First note that by integration by parts first in space and then integration in time we have

$$
I_{1}=-\frac{1}{2} \int_{t^{*}}^{T} \frac{d}{d t}\left\|\nabla \varphi_{1}(\cdot, t)\right\|^{2} \mathrm{~d} t=\frac{1}{2}\left\|\nabla \varphi_{1}\left(\cdot, t^{*}\right)\right\|^{2}-\frac{1}{2}\left\|\nabla \xi_{0}\right\|^{2} .
$$

The second term is handled using the decomposition of $u$ in the large scale and fine scale component and then an integration by parts only in the large scale part (the proof of this was detailed in [3], for completeness we reproduce the arguments in Appendix B). Let $\nabla_{S} u$ denote the symmetric part of the gradient of the vector $u$.

$$
\begin{aligned}
& I_{2}=-\left(\left(\nabla_{S} \bar{u}-\frac{1}{2}(\nabla \cdot \bar{u}) \mathcal{I}_{2 \times 2}\right) \nabla \varphi_{1}, \nabla \varphi_{1}\right)_{Q^{*}}-\left(u^{\prime} \cdot \nabla \varphi_{1}, \Delta \varphi_{1}\right)_{Q^{*}} \\
& \quad \leq \int_{Q}\left(\Lambda\left(\bar{u}, u^{\prime}, \nu\right) \nabla \varphi_{1}\right)^{\mathrm{T}} \cdot \nabla \varphi_{1} \mathrm{~d} x \mathrm{~d} t+\frac{1}{2}\left\|\nu^{\frac{1}{2}} \Delta \varphi_{1}\right\|_{Q^{*}}^{2} .
\end{aligned}
$$

In the second inequality we used Cauchy-Schwarz inequality followed by a geometricarithmetic inequality. Note that (4.10) holds for any decomposition $\bar{u}, u^{\prime}$. Since $u^{\prime}=u-\bar{u}$, it follows that we can minimize the first term on the right hand side over all large scale vector fields $\bar{u} \in L^{\infty}\left(0, t ; W^{1, \infty}(\Omega)\right)$ and the infimum value obtained is the inverse of the optimal timescale of the flow.

$$
\begin{array}{r}
\int_{Q}\left(\Lambda\left(\bar{u}, u^{\prime}, \nu\right) \nabla \varphi_{1}\right)^{\mathrm{T}} \cdot \nabla \varphi_{1} \mathrm{~d} x \mathrm{~d} t \leq \inf _{\bar{u} \in L^{\infty}\left(I ; W^{1, \infty}(\Omega)\right)}\left\|\sigma_{p}^{+}\left(\Lambda\left(\bar{u}, u^{\prime}, \nu\right)\right)\right\|_{L^{\infty}(Q)}\left\|\nabla \varphi_{1}\right\|_{Q}^{2} \\
=\tau_{F}^{-1}\left\|\nabla \varphi_{1}\right\|_{Q}^{2} .
\end{array}
$$


It is straightforward to see that

$$
\left(\tau_{F}\right)^{-1} \lesssim\|\nabla \bar{u}\|_{L^{\infty}(Q)}+\nu^{-1}\left\|u^{\prime}\right\|_{L^{\infty}(Q)}^{2}
$$

Therefore under the scale separation assumption discussed in the introduction, that there exists $\bar{u}$ with $\|\nabla \bar{u}\|_{L^{\infty}(Q)} \sim 1$ and $\nu^{-1}\left\|u^{\prime}\right\|_{L^{\infty}(Q)}^{2} \sim 1$, we have $\tau_{F} \sim 1$.

By an integration by parts and by using the relations $\nabla \cdot \operatorname{rot} \varphi=0$ and $\nabla \varphi \cdot \operatorname{rot} \varphi=$ 0 we have

$$
I_{3}=-\left(\omega_{h} \nabla \cdot \operatorname{rot} \varphi_{1}, \varphi_{1}\right)_{Q^{*}}-\left(\omega_{h} \operatorname{rot} \varphi_{1}, \nabla \varphi_{1}\right)_{Q^{*}}=0 .
$$

Collecting the results for $I_{1}-I_{3}$ in (4.9) we have

$$
\left\|\nabla \varphi_{1}\left(\cdot, t^{*}\right)\right\|^{2}+\left\|\nu^{\frac{1}{2}} \Delta \varphi_{1}\right\|_{Q^{*}}^{2} \lesssim \tau_{F}^{-1}\left\|\nabla \varphi_{1}\right\|_{Q^{*}}^{2}+\left\|\nabla \xi_{0}\right\|^{2} .
$$

The inequality for $\varphi_{1}$ follows after a Gronwall's inequality and by taking the supremum over $t^{*}$, resulting in

$$
\sup _{t \in I}\left\|\nabla \varphi_{1}(\cdot, t)\right\|^{2}+\left\|\nu^{\frac{1}{2}} D^{2} \varphi_{1}\right\|_{Q}^{2} \lesssim e^{\frac{T}{\tau_{F}}}\left\|\nabla \xi_{0}\right\|^{2} .
$$

Elliptic regularity has been used for the second term.

For the bound on $\varphi_{2}$, multiply equation (4.3) by $\varphi_{2}$ and integrate over $\Omega$,

$$
\left\|\nabla \varphi_{2}(\cdot, t)\right\|^{2}=-\left(\omega_{h} \operatorname{rot} \varphi_{1}, \nabla \varphi_{2}\right)_{\Omega} \leq\left\|\omega_{h}(\cdot, t)\right\|_{L^{\infty}(\Omega)}\left\|\nabla \varphi_{1}(\cdot, t)\right\|\left\|\nabla \varphi_{2}(\cdot, t)\right\| .
$$

Then divide by $\left\|\nabla \varphi_{2}(\cdot, t)\right\|$, integrate in time and use that

$$
\begin{aligned}
\int_{I}\left\|\nabla \varphi_{2}(\cdot, t)\right\| \mathrm{d} t \leq \int_{I}\left\|\omega_{h}(\cdot, t)\right\|_{L^{\infty}(\Omega)} & \left\|\nabla \varphi_{1}(\cdot, t)\right\| \mathrm{d} t \\
& \leq \int_{I}\left\|\omega_{h}(\cdot, t)\right\|_{L^{\infty}(\Omega)} \mathrm{d} t \sup _{t \in I}\left\|\nabla \varphi_{1}(\cdot, t)\right\| .
\end{aligned}
$$

Finally use equation (4.7) to bound $\sup _{t \in I}\left\|\nabla \varphi_{1}(\cdot, t)\right\|$.

Note the dependence on the $L^{\infty}$-norm of $\omega_{h}$ in the bound (4.8). This appearance of a finite element function in the stability estimate shows that the global stability depends on the monotonicity of the approximation scheme. However as we shall see, strict monotonicity is not necessary, only "sufficient" $L^{\infty}$-control of the discrete vorticity. We also point out that in case the Reynolds number is low the convection term $I_{2}$ may be treated using the inequality

$$
I_{2} \leq \frac{1}{2} \frac{U_{0}^{2} l}{l \nu}\left\|\nabla \varphi_{1}\right\|_{Q}^{2}+\frac{1}{2}\left\|\nu^{\frac{1}{2}} \Delta \varphi_{1}\right\|_{Q}^{2}
$$

where $l$ denotes the characteristic length scale of the flow, and $R e=\frac{U_{0} l}{\nu}<1$, resulting in exponential growth with the factor $e^{U_{0} l^{-1} T}$.

5. A posteriori error estimates for the abstract method. Let $e_{\omega}=\omega-\omega_{h}$ and define the filtered error $\tilde{e}_{\omega}:=\tilde{\omega}-\tilde{\omega}_{h}$, where a quantity $\tilde{v}$ is related to $v$ through the problem (with continuity of the traces of $\tilde{v}$ and $\nabla \tilde{v} \cdot n$ over the periodic boundaries)

$$
-\Delta \tilde{v}+\tilde{v}=v .
$$


We introduce a norm on $\tilde{e}_{\omega}$ such that $\left\|\tilde{e}_{\omega}\right\|_{-1}^{2}:=\left\|\nabla \tilde{e}_{\omega}\right\|^{2}+\left\|\tilde{e}_{\omega}\right\|^{2}=\left(e_{\omega}, \tilde{e}_{\omega}\right)_{\Omega}$. This norm is related to the $H^{-1}$-norm of $e_{\omega}$.

Using the above norm and the relations (3.14), (4.2)-(4.3) as well as the stability result of Proposition 4.1 we may derive a posteriori estimates for the filtered quantity $\tilde{e}_{\omega}$. We here derive the result for the abstract finite element method (3.3)-(3.4) and then show how these estimates can be transformed into a priori error estimates, depending on the properties of the stabilization operator $s\left(u_{h} ; \omega_{h}, v_{h}\right)$. The use of weak norms and stabilized finite element methods in the following estimates draws on ideas from [15] and [3]. Here the mesh parameters have been factored out, to stress the close relation between the a posteriori error estimates and the subsequent a priori error estimates. Using similar techniques one may derive estimates suitable for adaptivity where the mesh parameter is kept inside the residual expressions in order to associate mesh sizes to local residuals.

THEOREM 5.1. (A posteriori error estimates) Let $\omega, \omega_{h}$ be the solutions of (2.2)(2.3) and (3.3)-(3.4) respectively. Let $\tilde{\omega}, \tilde{\omega}_{h}$ be defined by the relation (5.1) and $\tau_{F}$ be defined by (4.5). Then there holds

$$
\left\|\left(\tilde{\omega}-\tilde{\omega}_{h}\right)(\cdot, T)\right\| \|_{-1} \lesssim e^{\frac{T}{\tau_{F}}} h^{\frac{1}{2}} \sum_{i=0}^{5} \mathcal{R}_{i},
$$

with

$$
\begin{gathered}
\mathcal{R}_{0}:=h^{\frac{1}{2}}\left\|\left(\omega-\omega_{h}\right)(\cdot, 0)\right\|, \\
\mathcal{R}_{1}:=\int_{I} \inf _{v_{h} \in V_{h}^{1}}\left\|h^{\frac{1}{2}}\left(u_{h} \cdot \nabla \omega_{h}-v_{h}\right)\right\| d t, \\
\mathcal{R}_{2}:=\min \left(h, \nu^{\frac{1}{2}} T^{\frac{1}{2}}\right)\left\|\nu^{\frac{1}{2}} \llbracket n_{F} \cdot \nabla \omega_{h} \rrbracket\right\|_{\mathcal{F} \times I}, \\
\mathcal{R}_{3}:=\int_{I}\left\|\omega_{h}(\cdot, t)\right\|_{L^{\infty}(\Omega)} d t \min \left(c_{0} \sup _{t \in I}\left\|\Psi_{h}(\cdot, t)\right\|_{\Delta, 0}, c_{1} h^{\frac{1}{2}} \sup _{t \in I}\left\|\omega_{h}(\cdot, t)\right\|\right)
\end{gathered}
$$

where

$$
\begin{gathered}
\left\|\Psi_{h}(\cdot, t)\right\|_{\Delta, s}:=\left\|h^{s} \llbracket n_{F} \cdot \nabla \Psi_{h}(\cdot, t) \rrbracket\right\|_{\mathcal{F}}+\inf _{v_{h} \in V_{h}^{l}}\left(\sum_{K \in \mathcal{T}_{h}}\left\|h^{\frac{1}{2}+s}\left(\Delta \Psi_{h}(\cdot, t)-v_{h}\right)\right\|_{K}^{2}\right)^{\frac{1}{2}}, \\
\mathcal{R}_{4}:=h^{\frac{3}{2}} \int_{I}\left\|\partial_{t} \nabla \omega_{h}\right\| d t
\end{gathered}
$$

and

$$
\mathcal{R}_{5}:=\left(U_{0}^{\frac{1}{2}}+U_{0}^{-\frac{1}{2}}\left\|u_{h}\right\|_{L^{\infty}(Q)}\right) \int_{I} s\left(u_{h} ; \omega_{h}, \omega_{h}\right)^{\frac{1}{2}} d t .
$$

The term $\mathcal{R}_{4}$ is omitted if the consistent mass matrix is used. For the velocities we have the estimate,

$$
\left\|\left(u-u_{h}\right)(\cdot, T)\right\| \lesssim\left(\left\|\Psi_{h}(\cdot, T)\right\|_{\Delta, \frac{1}{2}}+\left\|\left(\tilde{\omega}-\tilde{\omega}_{h}\right)(\cdot, T)\right\|_{-1}\right)
$$


where the second term in the right hand side may is a posteriori bounded by (5.2).

Proof. By the definition (5.1) of $\tilde{e}_{\omega}$ we have, taking $\xi_{0}=\tilde{e}_{\omega}$ in (4.4) and recalling that $e_{\Psi}:=\Psi-\Psi_{h}$

$$
\begin{aligned}
\left\|\tilde{e}_{\omega}(\cdot, T)\right\|_{-1}^{2}=( & \left.e_{\omega}(\cdot, T), \varphi_{1}(\cdot, T)\right)_{\Omega}+\left(e_{\omega},-\partial_{t} \varphi_{1}-u \cdot \nabla \varphi_{1}-\varphi_{2}-\nu \Delta \varphi_{1}\right)_{Q} \\
& \quad+\left(e_{\Psi},-\Delta \varphi_{2}-\nabla \omega_{h} \cdot \operatorname{rot} \varphi_{1}\right)_{Q} \\
= & \left(e_{\omega}(\cdot, 0), \varphi_{1}(\cdot, 0)\right)_{\Omega}+\left(\partial_{t} e_{\omega}+u \cdot \nabla e_{\omega}+\operatorname{rot} e_{\Psi} \cdot \nabla \omega_{h}, \varphi_{1}\right)_{Q} \\
& +\left(\nu \nabla e_{\omega}, \nabla \varphi_{1}\right)_{Q}+\left(\nabla e_{\Psi}, \nabla \varphi_{2}\right)_{Q}-\left(e_{\omega}, \varphi_{2}\right)_{Q} .
\end{aligned}
$$

Using now the consistency relation (3.14) and the $L^{2}$-orthogonality of the initial data we obtain

$$
\begin{aligned}
\left\|\tilde{e}_{\omega}(\cdot, T)\right\|_{-1}^{2}= & \left(e_{\omega}(\cdot, 0),\left(\varphi_{1}-\pi_{L} \varphi_{1}\right)(\cdot, 0)\right)_{\Omega} \\
& \quad+\left(\partial_{t} e_{\omega}+u \cdot \nabla e_{\omega}+\operatorname{rot} e_{\Psi} \cdot \nabla \omega_{h}, \varphi_{1}-\pi_{L} \varphi_{1}\right)_{Q} \\
+(\nu & \left.\nabla e_{\omega}, \nabla\left(\varphi_{1}-\pi_{L} \varphi_{1}\right)\right)_{Q}+\left(\nabla e_{\Psi}, \nabla\left(\varphi_{2}-\Pi \varphi_{2}\right)\right)_{Q}-\left(e_{\omega}, \varphi_{2}-\Pi \varphi_{2}\right)_{Q} \\
& +\int_{I}\left[\left(\partial_{t} \omega_{h}, \pi_{L} \varphi_{1}\right)_{\Omega}-m\left(\partial_{t} \omega_{h}, \pi_{L} \varphi_{1}\right)\right] \mathrm{d} t-s\left(u_{h} ; \omega_{h}, \pi_{L} \varphi_{1}\right),
\end{aligned}
$$

where $\Pi: H^{1}(\Omega) \mapsto V_{h}^{l}$ will be taken as either $\pi_{L}$ or $\pi_{V}$. Using the equations (2.2)(2.3) and the definitions of the projections $\pi_{L}$ and $\pi_{V}$ we deduce for $\Pi:=\pi_{V}$,

$$
\begin{gathered}
\left\|\tilde{e}_{\omega}(\cdot, T)\right\|_{-1}^{2}=\left(e_{\omega}(\cdot, 0),\left(\varphi_{1}-\pi_{L} \varphi_{1}\right)(\cdot, 0)\right)_{\Omega}-\left(u_{h} \cdot \nabla \omega_{h}-v_{h}, \varphi_{1}-\pi_{L} \varphi_{1}\right)_{Q} \\
-\left(\nu \nabla \omega_{h}, \nabla\left(\varphi_{1}-\pi_{L} \varphi_{1}\right)_{Q}+\left(\omega_{h}, \varphi_{2}-\pi_{V} \varphi_{2}\right)_{Q}\right. \\
+\int_{I}\left[\left(\partial_{t} \omega_{h}, \pi_{L} \varphi_{1}\right)_{\Omega}-m\left(\partial_{t} \omega_{h}, \pi_{L} \varphi_{1}\right)\right] \mathrm{d} t-\int_{I} s\left(u_{h} ; \omega_{h}, \pi_{L} \varphi_{1}\right) \mathrm{d} t, \forall v_{h}(t) \in V_{h}^{1},
\end{gathered}
$$

and similarly for $\Pi:=\pi_{L}$,

$$
\begin{gathered}
\left\|\tilde{e}_{\omega}(\cdot, T)\right\|_{-1}^{2}=\left(e_{\omega}(\cdot, 0),\left(\varphi_{1}-\pi_{L} \varphi_{1}\right)(\cdot, 0)\right)_{\Omega}-\left(u_{h} \cdot \nabla \omega_{h}-v_{h}, \varphi_{1}-\pi_{L} \varphi_{1}\right)_{Q} \\
-\left(\nu \nabla \omega_{h}, \nabla\left(\varphi_{1}-\pi_{L} \varphi_{1}\right)_{Q}-\left(\nabla \Psi_{h}, \nabla\left(\varphi_{2}-\pi_{L} \varphi_{2}\right)\right)_{Q}\right. \\
+\int_{I}\left[\left(\partial_{t} \omega_{h}, \pi_{L} \varphi_{1}\right)_{\Omega}-m\left(\partial_{t} \omega_{h}, \pi_{L} \varphi_{1}\right)\right] \mathrm{d} t-\int_{I} s\left(u_{h} ; \omega_{h}, \pi_{L} \varphi_{1}\right) \mathrm{d} t, \forall v_{h}(t) \in V_{h}^{1} .
\end{gathered}
$$

After some standard manipulation including integrations by parts, Cauchy-Schwarz inequalities, trace inequalities the approximation results (3.1) and (3.2), (3.11) and the stabilities (3.5) and (4.11) we may conclude, for $\Pi:=\pi_{V}$,

$$
\begin{aligned}
\left\|\tilde{e}_{\omega}(\cdot, T)\right\| \|_{-1}^{2} \lesssim h^{\frac{1}{2}}\left(h^{\frac{1}{2}}\left\|e_{\omega}(\cdot, 0)\right\|+\int_{I} \inf _{v_{h} \in V_{h}^{1}}\left\|h^{\frac{1}{2}}\left(u_{h} \cdot \nabla \omega_{h}-v_{h}\right)\right\| \mathrm{d} t\right. \\
+\min \left(h, \nu^{\frac{1}{2}} T^{\frac{1}{2}}\right)\left\|\nu^{\frac{1}{2}} \llbracket n_{F} \cdot \nabla \omega_{h} \rrbracket\right\|\left\|_{\mathcal{F} \times I}+c_{1} h^{\frac{1}{2}} \sup _{t \in I}\right\| \omega_{h}(\cdot, t)\left\|\int_{I}\right\| \omega_{h}(\cdot, t) \|_{L^{\infty}(\Omega)} \mathrm{d} t \\
\left.+h^{\frac{3}{2}} \int_{I}\left\|\partial_{t} \nabla \omega_{h}\right\| \mathrm{d} t+\left(U_{0}^{\frac{1}{2}}+U_{0}^{-\frac{1}{2}}\left\|u_{h}\right\|_{L^{\infty}(Q)}\right) \int_{I} s\left(u_{h} ; \omega_{h}, \omega_{h}\right)^{\frac{1}{2}} \mathrm{~d} t\right) \\
\quad \times\left(\sup _{t \in I}\left\|\nabla \varphi_{1}(\cdot, t)\right\|+\left\|\nu^{\frac{1}{2}} D^{2} \varphi_{1}\right\|_{Q}\right) .
\end{aligned}
$$

If $\Pi:=\pi_{L}$ the fourth term on the right hand side is replaced using

$$
\left(\nabla \Psi_{h}, \nabla\left(\varphi_{2}-\pi_{L} \varphi_{2}\right)\right)_{Q} \lesssim h^{\frac{1}{2}} c_{0} \sup _{t \in I}\left\|\Psi_{h}(t)\right\|_{\Delta, 0} \int_{I}\left\|\nabla \varphi_{2}(\cdot, t)\right\| \mathrm{d} t
$$


followed by the bound (4.8) on $\varphi_{2}$. The estimate (5.2) now follows by taking the minimum of the two expressions and noting that by (4.7)

$$
\sup _{t \in I}\left\|\nabla \varphi_{1}(\cdot, t)\right\|+\left\|\nu^{\frac{1}{2}} D^{2} \varphi_{1}\right\|_{Q} \lesssim e^{\frac{T}{\tau_{F}}}\left\|\tilde{e}_{\omega}\right\|_{-1} .
$$

The velocity estimate (5.3) is obtained by noting that there holds

$$
\left\|\left(u-u_{h}\right)(\cdot, T)\right\|^{2}:=\left\|\nabla e_{\Psi}(\cdot, T)\right\|^{2}=\left(\nabla e_{\Psi}, \nabla\left(e_{\Psi}-\pi_{L} e_{\Psi}\right)\right)_{\Omega}+\left(e_{\omega}, \pi_{L} e_{\Psi}\right)_{\Omega} .
$$

Using the equation (2.3) we have

$$
\begin{array}{r}
\left\|\nabla e_{\Psi}\right\|^{2}=-\left(\nabla \Psi_{h}, \nabla\left(e_{\Psi}-\pi_{L} e_{\Psi}\right)\right)_{\Omega}+\left(\omega, e_{\Psi}\right)_{\Omega}-\left(\omega_{h}, \pi_{L} e_{\Psi}\right)_{\Omega} \\
=-\left(\nabla \Psi_{h}, \nabla\left(e_{\Psi}-\pi_{L} e_{\Psi}\right)\right)_{\Omega}+\left(\omega-\omega_{h}, e_{\Psi}\right)_{\Omega} .
\end{array}
$$

Let $\tilde{e}$ be the solution of (5.1). Then

$$
\left\|u-u_{h}\right\|^{2}=-\left(\nabla \Psi_{h}, \nabla\left(e_{\Psi}-\pi_{L} e_{\Psi}\right)\right)_{\Omega}+\left(\nabla \tilde{e}_{\omega}, \nabla e_{\Psi}\right)_{\Omega}+\left(\tilde{e}_{\omega}, e_{\Psi}\right)_{\Omega} .
$$

By an integration by parts in the first term, followed by a Cauchy-Schwarz inequality and the Poincaré-Wirtinger inequality in the last term we may write

$$
\begin{aligned}
&\left\|u-u_{h}\right\|^{2} \lesssim\left\|h_{F}^{\frac{1}{2}} \llbracket \nabla \Psi_{h} \rrbracket\right\|_{\mathcal{F}}\left\|h_{F}^{-\frac{1}{2}}\left(e_{\Psi}-\pi_{L} e_{\Psi}\right)\right\|_{\mathcal{F}} \\
&+\left(\sum_{K \in \mathcal{T}_{h}}\left\|h\left(\Delta \Psi_{h}-v_{h}\right)\right\|_{K}^{2}\right)^{\frac{1}{2}}\left\|h^{-1}\left(e_{\Psi}-\pi_{L} e_{\Psi}\right)\right\| \\
&+\left\|\left(\tilde{\omega}-\tilde{\omega}_{h}\right)\right\|\left\|_{-1}\right\| u-u_{h} \| .
\end{aligned}
$$

By element wise trace inequalities and the approximation property (3.1) we have

$$
\left\|h^{-\frac{1}{2}}\left(e_{\Psi}-\pi_{L} e_{\Psi}\right)\right\|_{\mathcal{F}}+\left\|h^{-1}\left(e_{\Psi}-\pi_{L} e_{\Psi}\right)\right\| \lesssim\left\|u-u_{h}\right\|
$$

by which we conclude.

If the stability properties of the stabilized method are sufficient, these a posteriori error estimates translate into a priori error estimates. We propose two strategies for this. One based on Sobolev injections for discrete spaces and one based on monotonicity, applicable to monotone stabilized finite element methods. The advantage of the former is that it allows the use of the consistent mass matrix and (almost) linear stabilization terms. The latter technique on the other hand allows for the derivation of a priori error estimates with precise control of the constants in the estimates, but imposes the use of a diagonal mass matrix.

6. A priori error estimates for finite element methods using consistent mass matrix. In this section we consider the case of methods using a consistent mass-matrix, these methods will not be monotone, but do nevertheless allow for error estimates, using $L^{\infty}$-estimates on the vortcity that may be derived thanks to the stabilization.

Proposition 6.1. Assume that the meshes are quasi-uniform, that the mass matrix $m(\cdot, \cdot)$ is evaluated exactly and that in addition to (3.5) and (3.6) the following stability estimate holds for all $t>0$,

$$
\left\|\omega_{h}\right\|_{L^{\infty}(\Omega)} \lesssim c(h)\left(\left\|\omega_{h}\right\|+s\left(u_{h} ; \omega_{h}, \omega_{h}\right)^{\frac{1}{2}}\right) .
$$


Then there holds for all $\zeta>0$,

$$
\left\|\mid\left(\tilde{\omega}-\tilde{\omega}_{h}\right)(\cdot, T)\right\|_{-1} \lesssim C_{0} e^{\frac{T}{\tau_{F}}} h^{\frac{1}{2}}\left(c_{\zeta} h^{-\zeta}+c(h) h^{\frac{1}{2}}\right)
$$

and

$$
\left\|\left(u-u_{h}\right)(\cdot, T)\right\| \lesssim \inf _{\Phi_{h} \in V_{*}^{l}}\left\|\nabla\left(\Psi-\Phi_{h}\right)(\cdot, T)\right\|+C_{0} e^{\frac{T}{\tau_{F}}} h^{\frac{1}{2}}\left(c_{\zeta} h^{-\zeta}+c(h) h^{\frac{1}{2}}\right) .
$$

REMARK 6.1. For the above estimate to be useful we require $\zeta<\frac{1}{2}$ and $c(h) \sim h^{-\xi}$ with $\xi<1$. Typical values for these parameters obtained for the methods given in the next section are $\zeta=1 / 4$ and $c(h) \sim h^{-\xi}(1+|\log (h)|)$, with $\xi \in\{1 / 4,1 / 2\}$.

Proof. By (3.5) and (3.7) and by using a Cauchy-Schwarz inequality in time we bound $\mathcal{R}_{1}$,

$$
\int_{I} \inf _{v_{h} \in V_{h}^{1}}\left\|h^{\frac{1}{2}}\left(u_{h} \cdot \nabla \omega_{h}-v_{h}\right)\right\| \mathrm{d} t \lesssim U_{0}^{\frac{1}{2}} T^{\frac{1}{2}}\left(\int_{I} s\left(u_{h} ; \omega_{h}, \omega_{h}\right) \mathrm{d} t\right)^{\frac{1}{2}} \lesssim U_{0}^{\frac{1}{2}} T^{\frac{1}{2}}\|\omega(\cdot, 0)\| .
$$

Using an element wise trace inequality and (3.7) we also have for $\mathcal{R}_{2}$,

$$
\min \left(h, \nu^{\frac{1}{2}} T^{\frac{1}{2}}\right)\left\|\nu^{\frac{1}{2}} \llbracket n_{F} \cdot \nabla \omega_{h} \rrbracket\right\|_{\mathcal{F} \times I} \lesssim h^{\frac{1}{2}}\left\|\nu^{\frac{1}{2}} \nabla \omega_{h}\right\|_{Q} \lesssim h^{\frac{1}{2}}\|\omega(\cdot, 0)\| .
$$

For $\mathcal{R}_{3}$ we use (3.7) and the discrete Sobolev injection (6.1) to deduce

$$
\begin{aligned}
& \mathcal{R}_{3} \leq c_{1} h^{\frac{1}{2}} \sup _{t \in I}\left\|\omega_{h}(\cdot, t)\right\| \int_{I}\left\|\omega_{h}(\cdot, t)\right\|_{L^{\infty}(\Omega)} \mathrm{d} t \\
& \lesssim c_{1} h^{\frac{1}{2}}\|\omega(\cdot, 0)\| c(h) \int_{I}\left(\left\|\omega_{h}(\cdot, t)\right\|+s\left(u_{h} ; \omega_{h}, \omega_{h}\right)^{\frac{1}{2}}\right) \mathrm{d} t \\
& \lesssim c_{1} h^{\frac{1}{2}} c(h)\left(T+T^{\frac{1}{2}}\right)\|\omega(\cdot, 0)\|^{2} .
\end{aligned}
$$

The only remaining term is the stabilization term, $\mathcal{R}_{5}$ where in particular the factor $\left\|u_{h}\right\|_{L^{\infty}(Q)}$ needs to be bounded. Here we use (3.8) to deduce, for all $q>2$,

$$
\begin{aligned}
& U_{0}^{-\frac{1}{2}}\left\|u_{h}\right\|_{L^{\infty}(Q)} \int_{I} s\left(u_{h} ; \omega_{h}, \omega_{h}\right)^{\frac{1}{2}} \mathrm{~d} t \\
& \quad \leq c_{q} \sup _{t \in I}\left\|\omega_{h}(\cdot, t)\right\|_{L^{q}(\Omega)} U_{0}^{-\frac{1}{2}} T^{\frac{1}{2}}\left(\int_{I} s\left(u_{h} ; \omega_{h}, \omega_{h}\right) \mathrm{d} t\right)^{\frac{1}{2}}
\end{aligned}
$$

and by a global inverse inequality and the bound (3.7) we may conclude

$$
\begin{aligned}
U_{0}^{-\frac{1}{2}}\left\|u_{h}\right\|_{L^{\infty}(Q)} \int_{I} s\left(u_{h} ; \omega_{h}, \omega_{h}\right)^{\frac{1}{2}} \mathrm{~d} t \lesssim c_{q} h^{\frac{2-q}{q}} U_{0}^{-\frac{1}{2}} T^{\frac{1}{2}} \sup _{t \in I}\left\|\omega_{h}(\cdot, t)\right\|\|\omega(\cdot, 0)\| \\
\\
\leq c_{q} h^{\frac{2-q}{q}} U_{0}^{-\frac{1}{2}} T^{\frac{1}{2}}\|\omega(\cdot, 0)\|^{2}
\end{aligned}
$$

and the estimate follows taking $\zeta=(q-2) / q$. Note that the constant $c_{q}$ explodes as $q \rightarrow 2$. 
The bound in the $L^{2}$-norm for the velocities follows as before from the vorticity estimate. Let $\Phi_{h} \in V_{*}^{l}$. Then

$$
\begin{aligned}
& \left\|u-u_{h}\right\|^{2}=\left\|\nabla e_{\Psi}\right\|^{2}=\left(\nabla e_{\Psi}, \nabla\left(\Psi-\Phi_{h}\right)\right)_{\Omega}-\left(e_{\omega}, \Psi_{h}-\Phi_{h}\right)_{\Omega} \\
& \quad \leq\left\|u-u_{h}\right\|\left\|\nabla\left(\Psi-\Phi_{h}\right)\right\|-\left(-\Delta \tilde{e}_{\omega}+\tilde{e}_{\omega}, \Psi_{h}-\Phi_{h}\right)_{\Omega} \\
& \quad \leq\left\|u-u_{h}\right\|\left\|\nabla\left(\Psi-\Phi_{h}\right)\right\|+\left\|\tilde{\omega}-\tilde{\omega}_{h}\right\| \|_{-1}\left(\left\|\Psi-\Psi_{h}\right\|_{H^{1}(\Omega)}+\left\|\Psi-\Phi_{h}\right\|_{H^{1}(\Omega)}\right) \\
& \quad \leq C\left\|\nabla\left(\Psi-\Phi_{h}\right)\right\|^{2}+C\left\|\tilde{\omega}-\tilde{\omega}_{h}\right\|_{-1}^{2}+\frac{1}{2}\left\|u-u_{h}\right\|^{2}
\end{aligned}
$$

Where we used a Poincare-Wirtinger inequality followed by the arithmetic-geometric inequality with suitable weights, in the last inequality.

6.1. Examples of methods with consistent mass matrix satisfying the assumptions of Proposition 6.1. We consider first the stabilization method obtained by penalizing the jumps of the streamline derivative over element faces. We use the exact mass matrix in (3.3) and the stabilizing operator

$$
s_{s d}\left(u_{h} ; \omega_{h}, v_{h}\right):=\gamma \sum_{F \in \mathcal{F}} U_{0}^{-1}\left(h_{F}^{2} \llbracket u_{h} \cdot \nabla \omega_{h} \rrbracket, \llbracket u_{h} \cdot \nabla v_{h} \rrbracket\right)_{F} .
$$

For this formulation the following stability estimates hold

LEMMA 6.2. Assume that the consistent mass matrix is used in (3.3)-(3.4), then

$$
\sup _{t \in I}\left\|\omega_{h}(\cdot, t)\right\|^{2}+2\left\|\nu^{\frac{1}{2}} \nabla \omega_{h}\right\|_{Q}^{2}+2 \gamma U_{0}^{-1}\left\|h_{F} \llbracket u_{h} \cdot \nabla \omega_{h} \rrbracket\right\|_{\mathcal{F} \times I}^{2} \lesssim\|\omega(\cdot, 0)\|^{2}
$$

and if $l=1$,

$$
\left\|u_{h}(\cdot, T)\right\|^{2}+2\left\|\nu^{\frac{1}{2}}\left(\omega_{h}-\bar{\omega}_{h}\right)\right\|_{Q}^{2}=\left\|u_{h}(\cdot, 0)\right\|^{2},
$$

where

$$
\bar{\omega}_{h}:=\int_{\Omega} \omega_{h} d x
$$

Proof. the proof of (6.3) is an immediate consequence of (3.7) and the definition (6.2). The energy equality (6.4) follows by taking $v_{h}=\Psi_{h}$ in (3.3) and $\Phi_{h}=\nu\left(\omega_{h}-\right.$ $\left.\bar{\omega}_{h}\right)$ in (3.4) to obtain

$$
\int_{I} m\left(\partial_{t} \omega_{h}, \Psi_{h}\right) \mathrm{d} t+\left\|\nu^{\frac{1}{2}}\left(\omega_{h}-\bar{\omega}_{h}\right)\right\|_{Q}^{2}=-\int_{I} s_{s d}\left(u_{h} ; \omega_{h}, \Psi_{h}\right) \mathrm{d} t .
$$

The conclusion now follows by observing that by differentiating (3.4) in time we have

$$
m\left(\partial_{t} \omega_{h}, \Psi_{h}\right)=\left(\nabla \partial_{t} \Psi_{h}, \Psi_{h}\right)_{\Omega}=\frac{d}{d t}\left\|u_{h}(\cdot, t)\right\|^{2}
$$

and

$$
s_{s d}\left(u_{h} ; \omega_{h}, \Psi_{h}\right)=\gamma \sum_{F \in \mathcal{F}}\left(U_{0}^{-1} h_{F}^{2} \llbracket u_{h} \cdot \nabla \omega_{h} \rrbracket, \llbracket \operatorname{rot} \Psi_{h} \cdot \nabla \Psi_{h} \rrbracket\right)_{F}=0 .
$$


Observe that the stabilization in the method dissipates enstrophy but does not contribute to dissipation of energy. Using known results on interpolation between discrete spaces it is also straightforward to show (see [7]),

$$
U_{0}^{-\frac{1}{2}} \inf _{v_{h} \in V_{h}^{1}}\left\|h^{\frac{1}{2}}\left(u_{h} \cdot \nabla \omega_{h}-v_{h}\right)\right\|^{2} \lesssim s_{s d}\left(u_{h} ; \omega_{h}, \omega_{h}\right) .
$$

Unfortunately this stabilization operator can not be shown to satisfy (6.1). For this we need the stabilization to act also in the crosswind direction. We therefore propose the following two stabilization operators, the first is the standard artificial viscosity method

$$
s_{a v}\left(u_{h} ; \omega_{h}, v_{h}\right):=\left(\gamma h\left(U_{0}+\left|u_{h}\right|\right)^{2} U_{0}^{-1} \nabla \omega_{h}, \nabla v_{h}\right)
$$

and the second is a modification of (6.2) where also the crosswind gradient is penalized defined by

$$
s_{c d}\left(u_{h} ; \omega_{h}, v_{h}\right):=s_{s d}\left(u_{h} ; \omega_{h}, v_{h}\right)+\gamma_{1} \sum_{K \in \mathcal{F}} U_{0} h_{F}^{2} \int_{F} \llbracket n_{F} \cdot \nabla \omega_{h} \rrbracket \llbracket n_{F} \cdot \nabla v_{h} \rrbracket \mathrm{d} s .
$$

Observe that the first part of $s_{c d}$ ensures the satisfaction of (3.5) and as we shall see the second part is necessary for (6.1) to hold.

Proposition 6.3. Both stabilization operators (6.6) and (6.7) satisfy (3.5) and (3.6). The stabilization operator $s_{a v}(\cdot ; \cdot, \cdot)$ satisfies $(6.1)$ with $c(h) \sim h^{-\frac{1}{2}}(1+|\log (h)|)$ and $s_{c d}(\cdot ; \cdot, \cdot)$ satisfies $(6.1)$ with $c(h) \sim h^{-\frac{3}{4}}(1+|\log (h)|)$.

Proof. The proofs of (3.5) - (3.6) are consequences of the Cauchy-Schwarz inequality and in the case of $s_{c d}$ trace inequalities. To prove (6.1) we note that in two space dimensions there holds (see [21]),

$$
\left\|\omega_{h}\right\|_{L^{\infty}(\Omega)} \lesssim(1+|\log (h)|)\left\|\omega_{h}\right\|_{H^{1}(\Omega)}
$$

This allows us to conclude for $s_{a v}$. For $s_{c d}$ we first use the inequality (6.8) to bound the $L^{\infty}$-norm using the $H^{1}$ norm. We then need to prove that $s_{c d}$ gives sufficient control of the $H^{1}$-norm. Proceeding using integration by parts in $\int_{\Omega}\left|\nabla \omega_{h}\right|^{2} \mathrm{~d} x$, using that the gradient is constant on each element and taking absolute values in the integral we obtain the bound (see [7])

$$
\left\|\nabla \omega_{h}\right\| \leq\left(\sum_{F \in \mathcal{F}} \int_{F}\left|\llbracket n_{F} \cdot \nabla \omega_{h} \rrbracket\right|\left|\omega_{h}\right| \mathrm{d} s\right)^{\frac{1}{2}}
$$

A Cauchy-Schwarz inequality followed by a trace inequality in the right hand side leads to

$$
\left\|\nabla \omega_{h}\right\| \lesssim\left(\sum_{K \in \mathcal{T}_{h}} h^{-\frac{3}{2}}\left\|\omega_{h}\right\|_{K}\left\|h \llbracket n_{f} \cdot \nabla \omega_{h} \rrbracket\right\|_{\partial K}\right)^{\frac{1}{2}} \lesssim h^{-\frac{3}{4}}\left(U_{0}^{-\frac{1}{2}}\left\|\omega_{h}\right\|+s_{c d}\left(u_{h} ; \omega_{h}, \omega_{h}\right)^{\frac{1}{2}}\right) .
$$

We conclude that in this case

$$
\left\|\omega_{h}\right\|_{L^{\infty}(\Omega)} \lesssim(1+|\log (h)|) h^{-\frac{3}{4}}\left(U_{0}^{-\frac{1}{2}}\left\|\omega_{h}\right\|+s_{c d}\left(u_{h} ; \omega_{h}, \omega_{h}\right)^{\frac{1}{2}}\right) .
$$

Since the assumptions of Proposition 6.1 are satisfied, we may conclude that the 
method (3.3)-(3.4) using the stabilization (6.6) satisfies the a priori error bounds for $\zeta>0$

$$
\|\left.\left|\left(\tilde{\omega}-\tilde{\omega}_{h}\right)(\cdot, T)\right|\right|_{-1} \lesssim C_{0} e^{\frac{T}{\tau_{F}}} h^{\frac{1}{2}}\left(c_{\zeta} h^{-\zeta}+1+|\log (h)|\right)
$$

and

$$
\left\|\left(u-u_{h}\right)(\cdot, T)\right\| \lesssim \inf _{\Phi_{h} \in V_{*}^{l}}\left\|\nabla\left(\Psi-\Phi_{h}\right)(\cdot, T)\right\|+C_{0} e^{\frac{T}{\tau_{F}}} h^{\frac{1}{2}}\left(c_{\zeta} h^{-\zeta}+1+|\log (h)|\right) .
$$

Similarly we have the following estimates if the stabilization (6.7) is used.

$$
\left\|\mid\left(\tilde{\omega}-\tilde{\omega}_{h}\right)(\cdot, T)\right\|_{-1} \lesssim C_{0} e^{\frac{T}{\tau_{F}}} h^{\frac{1}{2}}\left(c_{\zeta} h^{-\zeta}+(1+|\log (h)|) h^{-\frac{1}{4}}\right)
$$

and

$$
\left\|\left(u-u_{h}\right)(\cdot, T)\right\| \lesssim \inf _{\Phi_{h} \in V_{*}^{l}}\left\|\nabla\left(\Psi-\Phi_{h}\right)(\cdot, T)\right\|+C_{0} e^{\frac{T}{\tau_{F}}} h^{\frac{1}{2}}\left(c_{\zeta} h^{-\zeta}+(1+|\log (h)|) h^{-\frac{1}{4}}\right) .
$$

We see that we obtain an a priori convergence order of $O\left(h^{\frac{1}{2}-\zeta}\right)$, for $\zeta>0$, when using (6.6), and $O\left(|\log (h)| h^{\frac{1}{4}}\right)$ for $(6.7)$, provided $\zeta<\frac{1}{4}$. Recalling that $\zeta=(q-2) / q$ we readily deduce that for $\zeta=1 / 4$ we have $q=8 / 3>2$ and hence this choice is valid. If $l=2$ the method using (6.7) has optimal convergence for smooth solutions by Proposition 3.2.

7. A priori error estimates for finite element methods using lumped mass matrix. Here we will assume that the finite element method satisfies a discrete maximum principle, in the sense that the bilinear form has the DMP-property. For details and theoretical background on these concepts we refer to $[4,5]$.

Proposition 7.1. (A priori error estimate using monotonicity) Assume that the meshes are quasi-uniform, that $l=1$, that $R e_{h}>1$, that the mass $m(\cdot, \cdot)$ is evaluated using nodal quadrature, that the form $a\left(u_{h} ; \omega_{h}, v_{h}\right)+s\left(u_{h} ; \omega_{h}, v_{h}\right)$ has the DMP property as defined in [5] and that (3.5)-(3.6) hold, Then

$$
\left\|\mid\left(\tilde{\omega}-\tilde{\omega}_{h}\right)(\cdot, T)\right\|_{-1} \lesssim C_{0} e^{\frac{T}{\tau F}} h^{\frac{1}{2}}
$$

and

$$
\left\|\left(u-u_{h}\right)(\cdot, T)\right\| \lesssim \inf _{\Phi_{h} \in V_{*}^{l}}\left\|\nabla\left(\Psi-\Phi_{h}\right)(\cdot, T)\right\|+C_{0} e^{\frac{T}{\tau_{F}}} h^{\frac{1}{2}}
$$

Proof. The terms $\mathcal{R}_{0}-\mathcal{R}_{2}$ are bounded as in the proof of Proposition 6.1. Since by assumption the spatial discretization of (3.3) has the DMP property and the massmatrix is evaluated using nodal quadrature, we know from $[4,5]$ that

$$
\left\|\omega_{h}\right\|_{L^{\infty}(Q)} \leq\left\|\omega_{h}(\cdot, 0)\right\|_{L^{\infty}(\Omega)} \lesssim\|\omega(\cdot, 0)\|_{L^{\infty}(\Omega)}
$$

Hence by $(3.8)\left\|u_{h}\right\|_{L^{\infty}(Q)} \lesssim\|\omega(\cdot, 0)\|_{L^{\infty}(\Omega)}$. We may then use these $L^{\infty}$-bounds together with the stabilities of Lemma 3.1 to upper bound the remaining residual quantities of (5.2). Using (3.8) and (3.7) we immediately have

$$
\mathcal{R}_{3} \leq h^{\frac{1}{2}} \sup _{t \in I}\left\|\omega_{h}(\cdot, t)\right\| \int_{I}\left\|\omega_{h}(\cdot, t)\right\|_{L^{\infty}(\Omega)} \mathrm{d} t \lesssim h^{\frac{1}{2}} T\left\|\omega_{h}(\cdot, 0)\right\|\left\|\omega_{h}(\cdot, 0)\right\|_{L^{\infty}(\Omega)}
$$


For the residual term $\mathcal{R}_{4}$ resulting from the mass lumping we observe that since $l=1$ the stability (3.9) holds. Using this result, the bound (3.8) together with the discrete maximum principle, the high Reynolds number assumption $U_{0} h>\nu$ and finally (3.7) we obtain

$$
\begin{aligned}
& \mathcal{R}_{4}=h^{\frac{3}{2}} \int_{I}\left\|\partial_{t} \nabla \omega_{h}\right\| \mathrm{d} t \\
& \lesssim T^{\frac{1}{2}}\left(\int_{I}\left(\left(U_{0}^{\frac{1}{2}}+U_{0}^{-\frac{1}{2}}\left\|u_{h}\right\|_{L^{\infty}(Q)}\right)^{2} s\left(u_{h} ; \omega_{h}, \omega_{h}\right)+U_{0}\left\|\nu^{\frac{1}{2}} \nabla \omega_{h}\right\|^{2}\right) \mathrm{d} t\right)^{\frac{1}{2}} \\
& \lesssim T^{\frac{1}{2}} U_{0}^{\frac{1}{2}}\|\omega(\cdot, 0)\| \text {. }
\end{aligned}
$$

The remaining contribution from the stabilization is bounded as before using the maximum principle and (3.7). The proof of the $L^{2}$-norm estimate on the velocities is identical to that of Proposition $6.1 \mathrm{\square}$

Note that only the proof of Proposition 7.1 uses the assumption $R e_{h}>1$ and only to control the non-consistent mass term. This constraint is likely to vanish if the method is analysed using techniques appropriate for parabolic problems, since mass lumping is known to be stable for dominant diffusion (see for instance [22]).

7.1. Examples of methods with lumped mass matrix satisfying the assumptions of Proposition 6.1. In this section we consider the case where the mass matrix is evaluated using nodal quadrature leading to a diagonal matrix. We also assume in this section that the meshes are Delaunay so that the discrete Laplace operator satisfies a discrete maximum principle, see [23]. Thanks to these two properties we will be able to design monotone finite element methods, that satisfy the assumptions of Proposition 7.1.

7.1.1. Linear artificial viscosity. A monotone method using linear artificial viscosity is obtained by taking (see [5])

$$
s\left(u_{h} ; \omega_{h}, v_{h}\right):=\gamma \sum_{K} U_{0} h_{K}^{2} \sum_{F \in \partial K}\left(\nabla \omega_{h} \times n_{F}, \nabla v_{h} \times n_{F}\right)_{F} .
$$

We may use the theory of $[4,5]$ to prove that the operator $a\left(\omega_{h}, v_{h}\right)+s\left(u_{h} ; \omega_{h}, v_{h}\right)$ has the DMP-property and hence the following discrete maximum principle holds

$$
\left\|\omega_{h}\right\|_{L^{\infty}(Q)} \leq\left\|\omega_{h}(\cdot, 0)\right\|_{L^{\infty}(\Omega)}
$$

This requires the parameter $\gamma$ to be chosen large enough, however it does not require any additional acute condition on the mesh, since the discretization of the Laplace operator results in an M-matrix on Delaunay meshes. Since by the maximum principle, $\left\|u_{h}\right\|_{L^{\infty}(Q)} \lesssim\left\|\omega_{h}(\cdot, 0)\right\|_{L^{\infty}(\Omega)}$ we have

$$
\left\|\left|u_{h}\right| h^{\frac{1}{2}} \nabla \omega_{h}\right\|_{Q}^{2} \lesssim\left\|u_{h}\right\|_{L^{\infty}(Q)}^{\frac{1}{2}} \int_{I} s\left(u_{h} ; \omega_{h}, \omega_{h}\right) \mathrm{d} t
$$

which proves (3.5) with $v_{h}=0$. It is straightforward to prove also (3.6). Comparing with Proposition 7.1 we conclude that the assumptions are satisfied and hence that the Proposition holds for (3.3)-(3.4) with stabilization given by (7.1) and the mass matrix evaluated using nodal quadrature. 
7.1.2. Nonlinear artificial viscosity. We propose a stabilization term consisting of one linear part and one nonlinear part. The role of the nonlinear part is to ensure that the form $a(\cdot ; \cdot, \cdot)+s(\cdot ; \cdot, \cdot)$ has the DMP property. The linear part is necessary to ensure that the inequality (3.5) holds. We define

$$
\begin{aligned}
s\left(u_{h} ; \omega_{h}, v_{h}\right) & :=s_{s d}\left(u_{h} ; \omega_{h}, v_{h}\right) \\
& +\gamma_{2} \sum_{K} h_{K}^{2} \sum_{F \in \partial K} R_{F}\left(\omega_{h}\right)\left(\operatorname{sign}\left(\nabla \omega_{h} \times n_{F}\right), \nabla v_{h} \times n_{F}\right)_{F}
\end{aligned}
$$

where

$$
R_{F}\left(\omega_{h}\right):=U_{0} m_{F}\left(\llbracket n_{F} \cdot \nabla \omega_{h} \rrbracket\right)
$$

with

$$
m_{F}\left(\llbracket n_{F} \cdot \nabla \omega_{h} \rrbracket\right)=\max _{\substack{F^{\prime} \in \mathcal{F} \\ F^{\prime} \in \partial K^{\prime} ; K^{\prime} \cap F=F}}\left|\llbracket n_{F^{\prime}} \cdot \nabla \omega_{h} \rrbracket\right| F_{F^{\prime}} \mid .
$$

It is shown in [5] that with this definition $a\left(u_{h} ; \omega_{h}, v_{h}\right)+s\left(u_{h} ; \omega_{h}, v_{h}\right)$ has the DMPproperty for $\gamma_{2}$ large enough and then (7.2) holds. To conclude we only need to show that the bounds (3.5) and (3.6) hold. From this the conclusions of Lemma 3.1 follows. Then the assumptions of Proposition 7.1 are satisfied and its estimates hold.

LEMma 7.2. The stabilization operator defined by (7.4) satisfies the bounds (3.5) and (3.6).

Proof. For the first inequality of (3.5) we observe that as before this holds thanks to the term $s_{s d}(\cdot ; \cdot, \cdot)$. The upper bound of (3.5) is also straightforward by repeated use of the trace inequality $h^{\frac{1}{2}}\left(\left\|n_{\partial K} \cdot \nabla \omega_{h}\right\|_{\partial K}+\left\|n_{\partial K} \times \nabla \omega_{h}\right\|_{\partial K}\right) \lesssim\left\|\nabla \omega_{h}\right\|_{K}$. Due to the lack of symmetry of the nonlinear operator the bound (3.6) requires a more detailed analysis. First note that

$$
\begin{aligned}
s\left(u_{h}, \omega_{h} ; v_{h}\right) \lesssim h^{\frac{1}{2}} U_{0}^{-\frac{1}{2}}\left\|u_{h}\right\|_{L^{\infty}(\Omega)} s_{s d}\left(u_{h} ; \omega_{h}, \omega_{h}\right)^{\frac{1}{2}}\left\|\nabla v_{h}\right\| & \\
& +\gamma_{2} \sum_{K} h_{K} \sum_{F \in \partial K}\left|R_{F}\left(\omega_{h}\right)\right|\left|\nabla v_{h}\right|_{F} \| F \mid .
\end{aligned}
$$

Considering the second term in the right hand side we may write

$$
\gamma_{2} \sum_{K} \sum_{F \in \partial K} h_{K}^{2}\left|R_{F}\left(\omega_{h}\right)\right|\left|\nabla v_{h}\right||F| \lesssim \gamma_{2}\left(\sum_{K} \sum_{F \in \partial K} h_{K}^{4}\left|R_{F}\left(\omega_{h}\right)\right|^{2}\right)^{\frac{1}{2}}\left\|\nabla v_{h}\right\| .
$$

It follows that we must prove the bound

$$
\gamma_{2} \sum_{K} \sum_{F \in \partial K} h_{K}^{4}\left|R_{F}\left(\omega_{h}\right)\right|^{2} \lesssim h U_{0} \gamma_{2} \sum_{K} h_{K}^{2} \sum_{F \in \partial K} R_{F}\left(\omega_{h}\right)\left\|\nabla \omega_{h} \times n_{F}\right\|_{L^{1}(F)} .
$$

For any face $F \in \mathcal{F}$ define $K_{F}^{+}$and $K_{F}^{-}$to be the triangles such that $K_{F}^{+} \cap K_{F}^{-}=F$. Assume that for a fixed face $F^{*}$ the max in $m_{F^{*}}$ is taken in the face $F^{\prime}$, associated to the elements $K_{F^{\prime}}^{+}$and $K_{F^{\prime}}^{-}$, so that

$$
m_{F^{*}}\left(\llbracket n_{F} \cdot \nabla \omega_{h} \rrbracket\right)=\left|\llbracket n_{F^{\prime}} \cdot \nabla \omega_{h} \rrbracket\right|_{F^{\prime}} \mid .
$$

Define the local set of faces $\mathcal{F}_{\Delta}^{\prime}:=\left\{F: F \in \partial K_{F^{\prime}}^{+} \cup \partial K_{F^{\prime}}^{-}\right\}$. Observing that

$$
\left|\llbracket n_{F^{\prime}} \cdot \nabla \omega_{h} \rrbracket\right|_{F^{\prime}} \mid h_{K} \lesssim\left\|\nabla \omega_{h} \times n_{F}\right\|_{L^{1}\left(\mathcal{F}_{\Delta}^{\prime}\right)}
$$


we may write

$$
\begin{aligned}
h_{K}\left|R_{F^{*}}\left(u_{h}, \omega_{h}\right)\right|^{2} \lesssim U_{0} R_{F^{*}}\left(u_{h}, \omega_{h}\right) & \left\|\nabla \omega_{h} \times n_{F}\right\|_{L^{1}\left(\mathcal{F}_{\Delta}^{\prime}\right)} \\
& \lesssim U_{0} \sum_{F \in \mathcal{F}_{\Delta}^{\prime}} R_{F}\left(u_{h}, \omega_{h}\right)\left\|\nabla \omega_{h} \times n_{F}\right\|_{L^{1}(F)} .
\end{aligned}
$$

The last inequality holds since the face $F^{\prime}$ that realises the max in $m_{F^{*}}$ is in the set over which the max in $m_{F}$ is taken for all $F \in \mathcal{F}_{\Delta}^{\prime}$ in the last expression. Applying now the inequality (7.7) to each term of the left hand side of (7.6), rearranging the sum, using the quasi-uniformity of the mesh and the upper bound on the cardinality of $\mathcal{F}_{\Delta}^{\prime}$ we conclude that (7.6) holds. Hence we have proven inequality (3.6).

8. Numerical examples. Below we will study two model problems. Our objective is to give numerical evidence of the following four points,

1. the exponential growth proportional to the gradient is indeed sharp and that the exponential instability is triggered by strong shear layers, i.e. strong layers in the velocity;

2. the convergence order of Proposition 3.2 is verified before instability sets in;

3. strong layers in the vorticity do not to give rise to exponential perturbation growth;

4. stabilization is necessary to get accurate results.

In all cases we considered the scheme (3.3)-(3.4) with the stabilization (6.7) and parameters $\gamma=0, \gamma_{1}=0.01$.

8.1. Kelvin-Helmholtz instability. We consider flow in the unit square with periodic boundary conditions in the $x$-direction and slip conditions at $y=0$ and $y=1$. The initial condition is chosen as

$$
\omega_{0}=-\frac{2}{\delta}\left(1-\tanh ((2 y-1) / \delta)^{2}\right)
$$

leading to the velocities

$$
u_{1}=\tanh ((2 y-1) / \delta), \quad u_{2}=0
$$

Here $\delta$ is a measure of the layer width and we let $\delta=1 /(7 L)$ with $L \in \mathbb{N}$. It follows that $\|u(\cdot, 0)\|_{W^{1, \infty}(\Omega)}=14 L$ and we expect the maximum gradient to be proportional to $L$. The initial vorticity $\omega_{0}$ is a stationary solution to the inviscid problem, but it is known to be unstable and lead to transition through the Kelvin-Helmholtz vortices. We consider a small kinematic viscosity $\nu=1 / 28 \cdot 10^{-4}$ and study the growth of $\left\|\omega_{h}(\cdot, 0)-\omega_{h}(\cdot, t)\right\|$, typically over 5000 timesteps of size $d t=7.8125 \cdot 10^{-4}$ for $L=$ $1,2,4$. This is a reasonable measure of the error since $\omega_{0}$ is a stationary solution to the inviscid problem and the diffusive effects are negligible for the viscosity and time-scales considered. Often when studying this problem an initial perturbation is added to reduce the dependence of the transition sequence on the mesh geometry (see for instance $[16,2]$.) Here we have not added any perturbation, so that the transition is an effect of perturbations induced by the discretization (in particular the use of penalty on the jump of the cross-wind gradient) and roundoff errors. Snapshots of the transition sequence for $L=2$ is presented in Figure 8.1. In the left plot of Figure 8.2 we show the perturbation growth for $L=1$ and $L=2$ on an $80 \times 80$ mesh and $L=4$ on an $160 \times 160$ mesh, with time step $d t / 2$. In these cases the perturbation growth is exponential with growth $e^{2 L t}$ when the transition takes place and hence the 


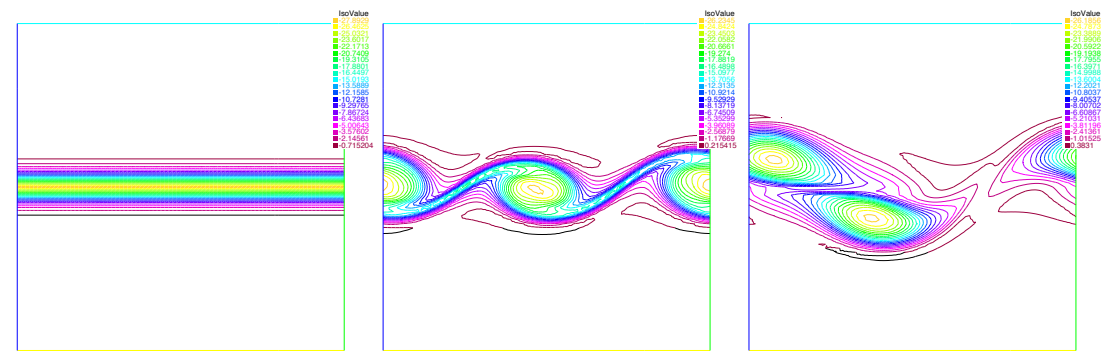

Figure 8.1. Kelvin-Helmholtz instability. Contour plots of the vorticity, for $L=2$, on the $80 \times 80$ mesh. From left to right, $T=0.7125, T=2.5$ and $T=3.21875$. Transition from the quasi-stationary initial data has taken place between $T=0.7125$ and $T=2.5$
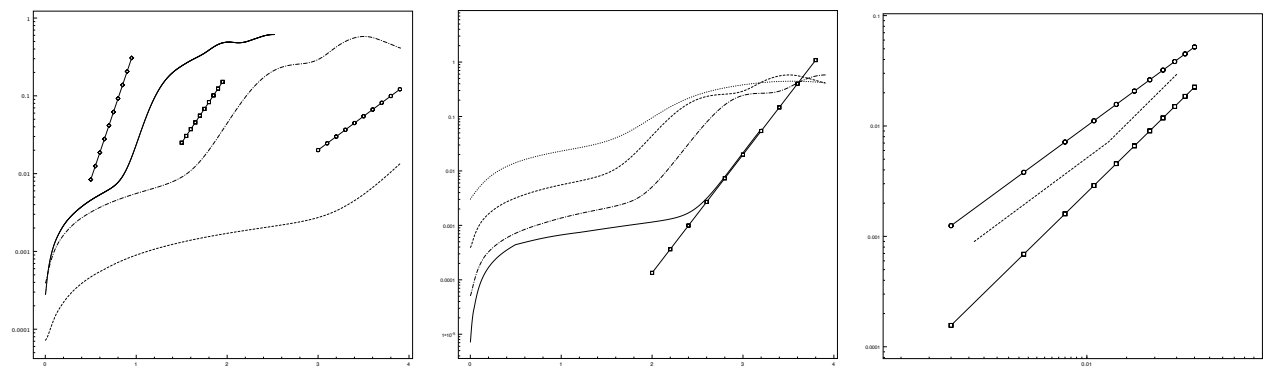

FIGURE 8.2. Kelvin-Helmholtz instability. Left: perturbation growth for different layer width, straight lines with markers give exponential growth $e^{2 L t}(L=1$ dashed/circles, $L=2$, dash dot/squares, $L=4$, filled/diamond). Center: perturbation growth for different refinement levels and $L=2(40 \times 40$ dotted, $80 \times 80$ dashed, $160 \times 160$ dash dot, $320 \times 320$ filled. Straight line with square marks $\left.e^{5 t}\right)$. Right: Convergence of the $L^{2}$-error for $L=2$ at $T=1$ indicated by the dashed line. Filled line with circles indicate $O\left(h^{\frac{3}{2}}\right)$ and with squares $O\left(h^{2}\right)$.

exponential coefficient is proportional to the maximum gradient. In the middle plot we show the perturbation growth for $L=2$ on four meshes, $40 \times 40,80 \times 80,160 \times 160$ and $320 \times 320$. On the finest mesh we used the smaller time-step size $d t / 2$. Observe that the exponential growth saturates on the $160 \times 160$ mesh, but is clearly smaller on the $40 \times 40$ mesh. We also observe that the initial time until transition increases with mesh refinement showing that as the initial perturbation becomes smaller it takes longer for it to trigger transition. In the right plot of Figure 8.2 we study the experimental convergence of $\left\|\omega_{h}(\cdot, 0)-\omega_{h}(\cdot, 1)\right\|$. Before transition we observe a rate between $O\left(h^{\frac{3}{2}}\right)$ and $O\left(h^{2}\right)$ as predicted by Proposition 3.2. 


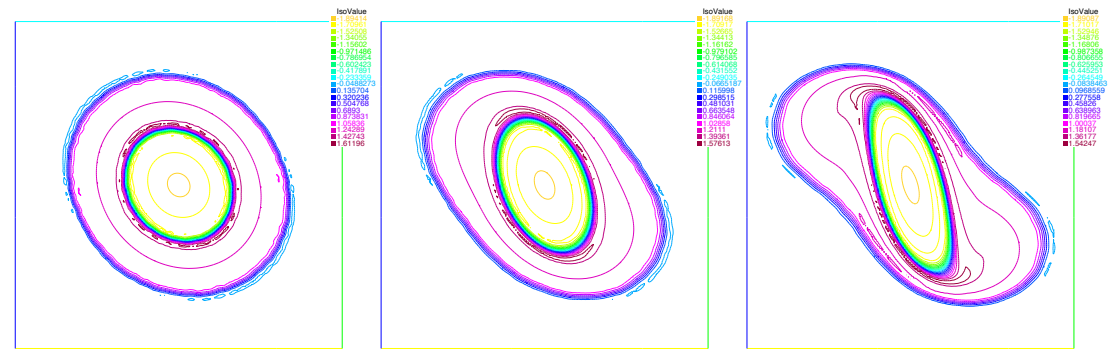

Figure 8.3. Discontinuous vorticity. Contour plots of the vorticity on the $80 \times 80$ mesh. From left to right, $T=0.7125, T=2.14375$ and $T=3.5625$

8.2. Discontinuous vorticity, inviscid case. The purpose of this example is to show the lack of sharpness of the estimate of Proposition 3.2, with the gradient of the vorticity in the exponential and the lack of robustness of the standard Galerkin method. Here we consider the case $\nu=0$ and consider the $\mathrm{x}$ - periodic domain $\left[-\frac{3}{2}, \frac{3}{2}\right]^{2}$, with non-penetration conditions at $y=-3 / 2$ and $y=3 / 2$. To reduce the influence of the mesh we have used a Union Jack type mesh and the initial vorticity was chosen as

$$
\omega_{0}=\left\{\begin{array}{cc}
-(2-r) & 0<r<\frac{1}{2} \\
2-r & \frac{1}{2}<r<1 \\
0 & 1<r
\end{array}\right.
$$

where $r=\sqrt{x^{2}+y^{2}}$. This vorticity is discontinuous at $r=\frac{1}{2}$ and at $r=1$. The resulting velocity field is a shear flow with a counter rotating vortex. This solution is not stationary, due to the boundary conditions on the domain. Instead the vortex is deformed by the shear flow as can be seen in the plots of Figure 8.3. We integrate the equations over the same time interval, with the same time-step, as in the previous example and as measure of the deformation we study once again the deviation from the inital data in the $L^{2}$-norm, $\left\|u_{h}(\cdot, 0)-u_{h}(\cdot, 1)\right\|$ (observing that this is not an error quantity.) We also compare with the result of a computation using the standard Galerkin method. In Figure 8.4 we give the evolution of the $L^{2}$-norm of the deviation from the initial data and we see that it grows linearly for the stabilized method, but blows up for the unstabilized method (we only present the standard Galerkin on the finest mesh, the coarse mesh solutions diverged even faster.) In a numerical experiment not presented here we extended the computational time by doubling the number of timestep, and did not observe any additional nonlinear effects, but the flow appeared to remain stable. 


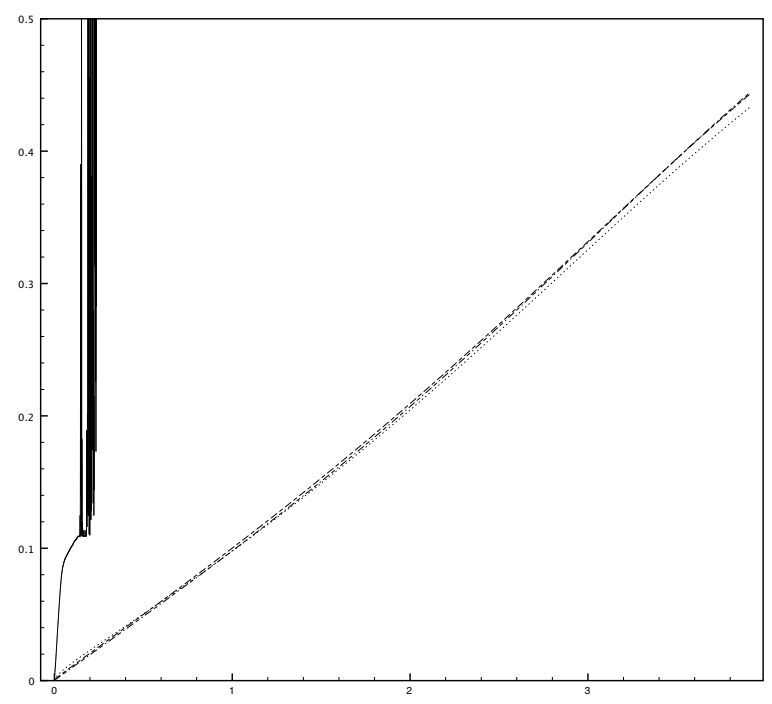

Figure 8.4. Discontinuous vorticity. Deviation from the initial data. Filled line standard Galerkin $160 \times 160$. Dotted line, stabilized $40 \times 40$. Dashed line, stabilized $80 \times 80$. Dash dotted line, stabilized $160 \times 160$.

9. Conclusion. We have derived error estimates for finite element approximations of the two dimensional Navier-Stokes' equations with a reduced stability factor that is independent of the finest scales of the flow. We have shown that, under a certain structural assumption on the solution of the two dimensional Navier-Stokes' equations, the estimates are robust with respect to the Reynolds number. The constant of the estimates depends on the $L^{\infty}$-norm of the initial vorticity and has exponential growth with exponential factor proportional to the gradient of the large scales of the flow. We considered error estimates in a weak norm for the the vorticity and in the $L^{2}$-norm for the velocities. Several stabilized finite element methods of first or second order accuracy for smooth solutions ares shown to enter the framework. If the solution is smooth we also prove that quasi optimal convergence may be obtained, provided the stabilization operator is weakly consistent to the right order.

Observe that in the low regularity case, even the large scales are assumed to have moderate gradients only, no $H^{2}$-regularity, so the convergence rates obtained are suboptimal either with $O\left(h^{\frac{1}{2}}\right)$ which is classical for stabilized finite element methods or, when no discrete maximum principle is satisfied, with $O\left(h^{\frac{3}{4}}\right)$.

It follows from the above analysis that if, for some quantity of interest, the adjoint perturbation equation has continuous dependence on inital data in the norm $\left.L^{\infty}\left(0, T ; H^{\frac{1}{2}+\varepsilon}\right)(\Omega)\right), \varepsilon>0$, independent of the Reynolds number, then this quantity may be computed using a stabilized finite element method. This observation of a general nature is true also for the velocity-pressure formulation and in three space dimensions.

Computational evidence in [14, Figure 1] shows that weaker control of the error, i.e. quantities of interest based on space and time averages, are expected to result in weaker growth in the dual problem and therefore more advantageous stability properties. 
Appendix A. Proof of Proposition 3.2. For simplicity we give the proof only in the inviscid case, $\nu=0$ and without crosswind stabilization, $\gamma_{1}=0$. The solution $\omega$ and $u$ is assumed to have moderate $H^{2}$-norm we also assume that $\nabla \omega$ is uniformly bounded. Consider the discrete errors

$$
e_{h, \Psi}:=\Psi_{h}-\pi_{V} \Psi \in V_{h}^{l} \text { and } e_{h, \omega}:=\omega_{h}-\pi_{L} \omega \in V_{h}^{1} .
$$

First consider the second equation (3.4) and use Galerkin orthogonality (second equation of (3.14))

$$
\left\|\nabla e_{h, \Psi}\right\|^{2}=\left(\nabla\left(\Psi-\pi_{V} \Psi\right), \nabla e_{h, \Psi}\right)_{\Omega}-\left(\omega-\omega_{h}, e_{h, \Psi}\right)_{\Omega}=-\left(\omega-\omega_{h}, e_{h, \Psi}\right)_{\Omega} .
$$

Applying a Cauchy-Schwarz inequality followed by a Poincaré inequality we obtain the following bound for $\Psi$ in terms of the error in the vorticity

$$
\left\|\nabla e_{h, \Psi}\right\| \lesssim\left\|\omega-\pi_{L} \omega_{h}\right\|+\left\|e_{h, \omega}\right\| .
$$

For the vorticity, first note that

$$
\frac{1}{2} \frac{\mathrm{d}}{\mathrm{dt}}\left\|e_{h, \omega}\right\|^{2}+s\left(u_{h} ; e_{h, \omega}, e_{h, \omega}\right)=m\left(\partial_{t} e_{h, \omega}, e_{h, \omega}\right)+\underbrace{a\left(u_{h} ; e_{h, \omega}, e_{h, \omega}\right)}_{=0}+s\left(u_{h} ; e_{h, \omega}, e_{h, \omega}\right) .
$$

Considering the first equation of (3.14) taking $v_{h}=e_{h, \omega}$ and there holds

$$
\begin{aligned}
\frac{1}{2} \frac{\mathrm{d}}{\mathrm{dt}}\left\|e_{h, \omega}\right\|^{2}+s\left(u_{h} ; e_{h, \omega}, e_{h, \omega}\right) & \\
=\left(\partial_{t}\left(\omega-\pi_{L} \omega\right), e_{h, \omega}\right)_{\Omega}+\left(\omega, u \cdot \nabla e_{h, \omega}\right)_{\Omega} & -\left(\pi_{L} \omega, u_{h} \cdot \nabla e_{h, \omega}\right)_{\Omega}-s\left(u_{h} ; \pi_{L} \omega, e_{h, \omega}\right) .
\end{aligned}
$$

By integration by parts in time we see that the first term on the right hand side is zero, by the orthogonality of the $L^{2}$-projection. We then add and subtract $u_{h}$ in the second term on the right hand side to obtain

$$
\begin{aligned}
\frac{1}{2} \frac{\mathrm{d}}{\mathrm{dt}}\left\|e_{h, \omega}\right\|^{2}+s\left(u_{h} ; e_{h, \omega}, e_{h, \omega}\right)=\left(\omega,\left(u-u_{h}\right) \cdot \nabla e_{h, \omega}\right)_{\Omega} \\
\quad+\left(\omega-\pi_{L} \omega, u_{h} \cdot \nabla e_{h, \omega}\right)_{\Omega}-s\left(u_{h} ; \pi_{L} \omega, e_{h, \omega}\right)=I+I I+I I I .
\end{aligned}
$$

In the first term on the right hand side we now reintegrate by parts and use CauchySchwarz inequality,

$$
\begin{aligned}
I & \leq\|\nabla \omega\|_{L^{\infty}(Q)}\left\|u-u_{h}\right\|\left\|e_{h, \omega}\right\| \\
& \lesssim\|\nabla \omega\|_{L^{\infty}(Q)}\left(\left\|\nabla\left(\Psi-\pi_{V} \Psi\right)\right\|^{2}+\left\|\omega-\pi_{L} \omega\right\|^{2}+\left\|e_{h, \omega}\right\|^{2}\right) .
\end{aligned}
$$

In the second term we use the orthogonality of the $L^{2}$-projection to retract some function $v_{h}$ and then apply (3.5),

$$
\begin{aligned}
I I=\left(\omega-\pi_{L} \omega, u_{h} \cdot \nabla e_{h, \omega}-v_{h}\right)_{\Omega} & \leq c U_{0}^{\frac{1}{2}}\left\|h^{-\frac{1}{2}}\left(\omega-\pi_{L} \omega\right)\right\| s\left(u_{h} ; e_{h, \omega}, e_{h, \omega}\right)^{\frac{1}{2}} \\
& \leq c h^{3} U_{0}\|\omega\|_{H^{2}(\Omega)}^{2}+\frac{1}{4} s\left(u_{h} ; e_{h, \omega}, e_{h, \omega}\right) .
\end{aligned}
$$

For the stabilization term finally we apply the Cauchy-Schwarz inequality and an arithmetic-geometric inequality to obtain

$$
I I I=s\left(u_{h} ; \pi_{L} \omega, e_{h, \omega}\right) \leq s\left(u_{h} ; \pi_{L} \omega, \pi_{L} \omega\right)+\frac{1}{4} s\left(u_{h} ; e_{h, \omega}, e_{h, \omega}\right) .
$$


Then we observe that by adding and subtracting $\operatorname{rot} \pi_{V} \Psi$ we may write

$$
s\left(u_{h} ; \pi_{L} \omega, \pi_{L} \omega\right) \lesssim s\left(u_{h}-\operatorname{rot} \pi_{V} \Psi ; \pi_{L} \omega, \pi_{L} \omega\right)+s\left(\operatorname{rot} \pi_{V} \Psi ; \pi_{L} \omega, \pi_{L} \omega\right) .
$$

Using the definition (6.2) and the stability of the $L^{2}$-projection on quasi uniform meshes, we have,

$$
\begin{aligned}
s\left(u_{h}-\operatorname{rot} \pi_{V} \Psi ; \pi_{L} \omega, \pi_{L} \omega\right) & \lesssim U_{0}^{-1} \sum_{F \in \mathcal{F}} \int_{F} h^{2}\left|\nabla e_{h, \Psi}\right|^{2}\left|\nabla \pi_{L} \omega_{h}\right|^{2} \mathrm{~d} s \\
& \lesssim U_{0}^{-1}\|\nabla \omega\|_{L^{\infty}(\Omega)}^{2} h\left\|\nabla e_{h, \Psi}\right\|^{2} \\
& \lesssim U_{0}^{-1}\|\nabla \omega\|_{L^{\infty}(\Omega)}^{2} h\left(\left\|\omega-\pi_{L} \omega\right\|^{2}+\left\|e_{h, \omega}\right\|^{2}\right)
\end{aligned}
$$

and then, once again using trace inequalities,

$$
\begin{aligned}
s\left(\operatorname{rot} \pi_{V} \Psi ;\right. & \left.\pi_{L} \omega, \pi_{L} \omega\right) \\
& \leq U_{0}^{-1}\left\|\nabla \pi_{V} \Psi\right\|_{L^{\infty}(Q)}^{2} \sum_{K} h_{K}\left(\left\|\nabla\left(\omega-\pi_{L} \omega\right)\right\|_{K}^{2}+h_{K}^{2}\left\|\nabla\left(\omega-\pi_{L} \omega\right)\right\|^{2}\right) \\
& \leq U_{0}^{-1}\|u\|_{L^{\infty}(\Omega)}^{2} C h^{3}\|\omega\|_{H^{2}(\Omega)}^{2} .
\end{aligned}
$$

We conclude by collecting the upper bounds for the terms $I-I I I$, applying approximability, integrating in time and using Gronwall's lemma, that

$$
\begin{aligned}
\left\|e_{h, \omega}(\cdot, T)\right\|^{2} & +\int_{I} s\left(u_{h} ; e_{h, \omega}, e_{h, \omega}\right) \lesssim \exp \left(c T\|\nabla \omega\|_{L^{\infty}(Q)}\right)\|\nabla \omega\|_{L^{\infty}(Q)} \\
& \times\left(h^{3}\|\omega\|_{L^{2}\left(I ; H^{2}(\Omega)\right)}^{2}+h^{2 l}\|\Psi\|_{L^{2}\left(I ; H^{l+1}(\Omega)\right)}^{2}\right)
\end{aligned}
$$

Here we assumed $h\|\nabla \omega\|_{L^{\infty}(Q)} \lesssim 1$ and neglected the dependence on $U_{0}$ and $\|u\|_{L^{\infty}(Q)}^{2}$ (that is upper bounded by $\left.\|\omega(\cdot, 0)\|_{L^{\infty}(\Omega)}^{2}\right)$. The bound on $\left\|\nabla\left(\pi_{V} \Psi-\Psi_{h}\right)\right\|$ is immediate from (A.2) and the bound on $s\left(u_{h} ; \omega_{h}, \omega_{h}\right)$ follows the triangle inequality

$$
s\left(u_{h} ; \omega_{h}, \omega_{h}\right) \lesssim s\left(u_{h} ; \pi_{L} \omega, \pi_{L} \omega\right)+s\left(u_{h} ; e_{h, \omega}, e_{h, \omega}\right)
$$

and the bounds (A.4)-(A.5) and (A.6).

Appendix B. Proof of the bound (4.10). First split the velocity field in the large and the fine scale component,

$$
I_{2}=\left(u \cdot \nabla \varphi_{1}, \Delta \varphi_{1}\right)_{Q^{*}}=\left(\bar{u} \cdot \nabla \varphi_{1}, \Delta \varphi_{1}\right)_{Q^{*}}+\left(u^{\prime} \cdot \nabla \varphi_{1}, \Delta \varphi_{1}\right)_{Q^{*}},
$$

then integrate by parts in the term representing the large scale transport. Recalling that $\varphi_{1} \in L^{2}\left(I ; H^{2}(\Omega)\right)$ and $\bar{u} \in L^{\infty}\left(I ; W^{1, \infty}(\Omega)\right)$ we have $\bar{u} \cdot \nabla \varphi_{1} \in L^{2}\left(I ; H_{p e r}^{1}(\Omega)\right)$. Then the following integration by parts is justified

$$
\left(\bar{u} \cdot \nabla \varphi_{1}, \Delta \varphi_{1}\right)_{Q^{*}}=-\left(\nabla\left(\bar{u} \cdot \nabla \varphi_{1}\right), \nabla \varphi_{1}\right)_{Q^{*}} .
$$

Note that by the product rule

$$
\left(\nabla\left(\bar{u} \cdot \nabla \varphi_{1}\right), \nabla \varphi_{1}\right)_{Q^{*}}=\sum_{i=1}^{d}\left(\left(\partial_{x_{i}} \bar{u}\right) \cdot \nabla \varphi_{1}, \partial_{x_{i}} \varphi_{1}\right)_{Q^{*}}+\sum_{i=1}^{d}\left(\bar{u} \cdot\left(\partial_{x_{i}} \nabla \varphi_{1}\right), \partial_{x_{i}} \varphi_{1}\right)_{Q^{*}}
$$


For the first sum of the right hand side we have

$$
\sum_{i=1}^{d}\left(\left(\partial_{x_{i}} \bar{u}\right) \cdot \nabla \varphi_{1}, \partial_{x_{i}} \varphi_{1}\right)_{Q^{*}}=\left(\left(\nabla_{S} \bar{u}\right) \nabla \varphi_{1}, \nabla \varphi_{1}\right)_{Q^{*}}
$$

where $\nabla_{S}$ denotes the symmetric part of the gradient tensor. Similarly we obtain for the second part

$$
\begin{aligned}
\sum_{i=1}^{d}\left(\bar{u} \cdot\left(\partial_{x_{i}} \nabla \varphi_{1}\right), \partial_{x_{i}} \varphi_{1}\right)_{Q^{*}}=\sum_{i=1}^{d} \sum_{j=1}^{d}\left(\bar{u}_{j}\left(\partial_{x_{i}} \partial_{x_{j}} \varphi_{1}\right), \partial_{x_{i}} \varphi_{1}\right)_{Q^{*}} \\
=\sum_{i=1}^{d} \sum_{j=1}^{d}\left(\bar{u}_{j}\left(\partial_{x_{j}} \partial_{x_{i}} \varphi_{1}\right), \partial_{x_{i}} \varphi_{1}\right)_{Q^{*}}=\sum_{i=1}^{d}\left(\bar{u} \cdot \nabla \partial_{x_{i}} \varphi_{1}, \partial_{x_{i}} \varphi_{1}\right)_{Q^{*}}
\end{aligned}
$$

By the divergence theorem we then have

$$
\sum_{i=1}^{d}\left(\bar{u} \cdot \nabla \partial_{x_{i}} \varphi_{1}, \partial_{x_{i}} \varphi_{1}\right)_{Q^{*}}=-\frac{1}{2} \sum_{i=1}^{d}\left(\nabla \cdot \bar{u} \partial_{x_{i}} \varphi_{1}, \partial_{x_{i}} \varphi_{1}\right)_{Q^{*}}
$$

We conclude that, with $\mathcal{I}_{2 \times 2}$ denoting the identity matrix,

$$
\left(\bar{u} \cdot \nabla \varphi_{1}, \Delta \varphi_{1}\right)_{Q^{*}}=\left(\left(-\nabla_{S} \bar{u}+\frac{1}{2} \nabla \cdot \bar{u} \mathcal{I}_{2 \times 2}\right) \nabla \varphi_{1}, \nabla \varphi_{1}\right)_{Q^{*}}
$$

Observing that

$$
\begin{aligned}
\left(u^{\prime} \cdot \nabla \varphi_{1}, \Delta \varphi_{1}\right)_{Q^{*}} \leq \| \nu^{-1 / 2} u^{\prime} \cdot \nabla \varphi_{1} & \left\|_{Q^{*}}\right\| \nu^{\frac{1}{2}} \Delta \varphi_{1} \|_{Q^{*}} \\
& \leq \frac{1}{2}\left\|\nu^{-1 / 2} u^{\prime} \cdot \nabla \varphi_{1}\right\|_{Q^{*}}^{2}+\frac{1}{2}\left\|\nu^{\frac{1}{2}} \Delta \varphi_{1}\right\|_{Q^{*}}^{2}
\end{aligned}
$$

we have,

$$
I_{2} \leq \int_{Q^{*}}\left(\Lambda\left(\bar{u}, u^{\prime}, \nu\right) \nabla \varphi_{1}\right)^{\mathrm{T}} \cdot \nabla \varphi_{1} \mathrm{~d} x \mathrm{~d} t+\frac{1}{2}\left\|\nu^{\frac{1}{2}} \Delta \varphi_{1}\right\|_{Q^{*}}^{2},
$$

where

$$
\Lambda\left(\bar{u}, u^{\prime}, \nu\right):=-\nabla_{S} \bar{u}+\frac{1}{2} \nabla \cdot \bar{u} \mathcal{I}_{2 \times 2}+\frac{1}{2} u^{\prime T} u^{\prime} \nu^{-1}
$$

\section{REFERENCES}

[1] R. Bank and $\mathrm{H}$ Yserentant. On the $H^{1}$-stability of the $L_{2}$-projection onto finite element spaces. Numerische Mathematik, 126(2):361-381, 2014.

[2] E. Burman. Interior penalty variational multiscale method for the incompressible Navier-Stokes equation: monitoring artificial dissipation. Comput. Methods Appl. Mech. Engrg., 196(4144):4045-4058, 2007.

[3] E. Burman. Robust error estimates in weak norms for advection dominated transport problems with rough data. Maths. Mod. Meths. App. Sci., 2014. online ready, doi:10.1142/S021820251450033X.

[4] E. Burman and A. Ern. The discrete maximum principle for stabilized finite element methods. In Franco Brezzi, Annalisa Buffa, Stefania Corsaro, and Almerico Murli, editors, Numerical Mathematics and Advanced Applications, pages 557-566. Springer Milan, 2003. 
[5] E. Burman and A. Ern. Stabilized Galerkin approximation of convection-diffusion-reaction equations: discrete maximum principle and convergence. Math. Comp., 74(252):1637-1652 (electronic), 2005.

[6] E. Burman and M. A. Fernández. Continuous interior penalty finite element method for the time-dependent Navier-Stokes equations: space discretization and convergence. Numer. Math., 107(1):39-77, 2007.

[7] E. Burman and P. Hansbo. Edge stabilization for Galerkin approximations of convectiondiffusion-reaction problems. Comput. Methods Appl. Mech. Engrg., 193(15-16):1437-1453, 2004.

[8] A. Demlow, D. Leykekhman, A. H. Schatz, and L. B. Wahlbin. Best approximation property in the $W_{\infty}^{1}$ norm for finite element methods on graded meshes. Math. Comp., 81(278):743$764,2012$.

[9] A. Dunca and V. John. Finite element error analysis of space averaged flow fields defined by a differential filter. Math. Models Methods Appl. Sci., 14(4):603-618, 2004.

[10] A. Dunca, V. John, and W. Layton. Approximating local averages of fluid velocities: The equilibrium Navier-Stokes equations. Appl. Numer. Math., 49(2):187-205, 2004.

[11] C. Foias, O. Manley, and R. Temam. Modelling of the interaction of small and large eddies in two-dimensional turbulent flows. RAIRO Modél. Math. Anal. Numér., 22(1):93-118, 1988.

[12] P. Hansbo and A. Szepessy. A velocity-pressure streamline diffusion finite element method for the incompressible Navier-Stokes equations. Comput. Methods Appl. Mech. Engrg., 84(2):175-192, 1990.

[13] A. A. Himonas and G. Misiołek. Non-uniform dependence on initial data of solutions to the Euler equations of hydrodynamics. Comm. Math. Phys., 296(1):285-301, 2010.

[14] J. Hoffman and C. Johnson. Stability of the dual Navier-Stokes equations and efficient computation of mean output in turbulent flow using adaptive DNS/LES. Comput. Methods Appl. Mech. Engrg., 195(13-16):1709-1721, 2006.

[15] P. Houston, J. A. Mackenzie, E. Süli, and G. Warnecke. A posteriori error analysis for numerical approximations of Friedrichs systems. Numer. Math., 82(3):433-470, 1999.

[16] V. John. An assessment of two models for the subgrid scale tensor in the rational LES model. J. Comput. Appl. Math., 173(1):57-80, 2005.

[17] C. Johnson and J. Saranen. Streamline diffusion methods for the incompressible Euler and Navier-Stokes equations. Math. Comp., 47(175):1-18, 1986.

[18] J.-G. Liu and C.-W. Shu. A high-order discontinuous Galerkin method for 2D incompressible flows. J. Comput. Phys., 160(2):577-596, 2000.

[19] V. Maz'ya. On the boundedness of first derivatives for solutions to the Neumann-Laplace problem in a convex domain. J. Math. Sci. (N. Y.), 159(1):104-112, 2009. Problems in mathematical analysis. No. 40.

[20] R. Rannacher and R. Scott. Some optimal error estimates for piecewise linear finite element approximations. Math. Comp., 38(158):437-445, 1982.

[21] R. Scott. Optimal $L^{\infty}$ estimates for the finite element method on irregular meshes. Math. Comp., 30(136):681-697, 1976.

[22] V. Thomée. Galerkin finite element methods for parabolic problems, volume 25 of Springer Series in Computational Mathematics. Springer-Verlag, Berlin, 1997.

[23] J. Xu and L. Zikatanov. A monotone finite element scheme for convection-diffusion equations. Math. Comp., 68(228):1429-1446, 1999. 